\title{
Efecto de la ganadería y la variación estacional sobre la composición florística y la biomasa vegetal en los humedales de la costa centro oeste del Golfo de México
}

\section{Effect of livestock and seasonal variation on floristic composition and plant biomass in wetlands of the central western coast of the Gulf of Mexico}

\author{
Karla Rodríguez-Medinal (D, Patricia Moreno-Casasola1,3 (D), Carlos Yañez-Arenas² (1)
}

\begin{abstract}
1 Instituto de Ecología, A.C., Red de Ecología Funcional, Carretera antigua a Coatepec 351, El Haya, 91070 Xalapa, Veracruz, México.

2 Universidad Nacional Autónoma de México, Facultad de Ciencias, Parque Científico y Tecnológico de Yucatán Laboratorio de Conservación de la Biodiversidad. Km. 5.5 Carr. Sierra Papacal-Chuburná Puerto, Sierra Papacal, 97302 Mérida, Yucatan, México.

3 Autor para la correspondencia: patricia.moreno@inecol.mx
\end{abstract}

Citar como:

Rodríguez-Medina, K., P. Moreno-Casasola y C. Yañez-Arenas. 2017. Efecto de la ganadería y la variación estacional sobre la composición florística y la biomasa vegetal en los humedales de la costa centro oeste del Golfo de México. Acta Botanica Mexicana 119: 79-99. DOI: http://dx.doi.org/10.21829/ abm119. 2017.1233

Recibido: 28 de enero de 2016 Revisado: 22 de agosto de 2016 Aceptado: 11 de noviembre de 2016.

DOI

http://dx.doi.org/10.21829/abml19. 2017.1233
Resumen:

Antecedentes y Objetivos: Los humedales son ecosistemas altamente productivos que aportan diversos servicios ecosistémicos; sin embargo, también son de los más amenazados debido a actividades humanas como la agricultura y ganadería. México ha perdido $62 \%$ de sus humedales y específicamente los de la costa central del Golfo de México se están viendo afectados debido a la actividad ganadera. Existen pocos trabajos acerca del tema; por tanto, en esta investigación se analizó el efecto de la ganadería y la estacionalidad sobre la composición florística, biomasa aérea (BA) y biomasa subterránea (BS) de humedales herbáceos de la laguna de Alvarado, Veracruz, México.

Métodos: Se seleccionaron dos localidades de trabajo y en cada una se eligieron dos sitios de muestreo. En cada sitio se colocaron ocho parcelas de $1 \mathrm{~m}^{2}$ (la mitad fueron cercadas para evitar al ganado). Durante un año se obtuvieron datos de riqueza y diversidad de especies, cobertura vegetal, BA y BS. Con estos datos se calculó el valor de importancia relativa (VIR) por especie e índice de reemplazo de especies.

Resultados clave: Se registraron 29 especies y se observó un gradiente de perturbación, resultando las localidades de Río Blanco las más conservadas. En general la riqueza fue significativamente mayor en lluvias y las especies con mayor VIR fueron las propias de humedales, aunque hubo gramíneas dominantes en ambas temporadas. La BS fue significativamente mayor en Río Blanco (sitio 1), y la BA en Río Blanco 1 y Río Limón 1. En lluvias la BA fue mayor en la mitad de los sitios.

Conclusiones: Con los resultados de este trabajo se observa que los humedales herbáceos de agua dulce pueden conservar sus funciones ecológicas en presencia de la ganadería siempre y cuando sea de bajo impacto. Inclusive, si ésta se practica de manera controlada, puede favorecer la diversidad de especies nativas del humedal y limitar el crecimiento de pastos invasores.

Palabras clave: especies invasoras, impacto humano, inundación, popales, riqueza florística.

\section{ABSTRACT:}

Background and Aims: Wetlands are highly productive ecosystems, which provide many environmental services. However, they are one of the most threatened due to human activities such as agriculture and livestock. Mexico has lost $62 \%$ of its wetlands and specifically those of the central coast of the Gulf of Mexico are being affected due to livestock pressure. There are very few studies about the subject, so in this paper we analyze the effect of livestock and seasonality on floristic composition, above ground biomass (AB) and below ground biomass (BB) of the Alvarado lagoon, Veracruz, Mexico.

Methods: We selected two working locations, and in each locality chose two sampling sites. In each site, we placed eight $1 \mathrm{~m}^{2}$ plots of four different locations (half were fenced to keep out livestock). For an entire year we obtained species richness and diversity, and data on vegetation cover, as well as $\mathrm{AB}$ and BB. With the data obtained, we calculated the Relative Importance Value (RIV) by species and the relative replacement rate of species.

Key results: Total richness resulted in 29 species. A disturbance gradient was observed, with the best conserved sites in Río Blanco. In most sites, species richness was significantly higher in rainfall and species with high RIV were typical wetland species, although there were dominant grasses in both climatic seasons. $\mathrm{BB}$ was significantly higher in one location of Rio Blanco, and $\mathrm{AB}$ in one location of Rio Blanco and in one from Rio Limon. During the rainy season, $\mathrm{AB}$ was higher in half of the sites.

Conclusions: We conclude that freshwater wetlands can retain their ecological functions in the presence of livestock as long as the impact is low. Even if there is cattle grazing with a controlled management, it can promote diversity of native wetland species and limit the growth of invasive grasses.

Key words: flood, herbaceous wetlands, human impact, invasive species, species richness. 


\section{INTRODUCCIÓN}

Los humedales son ecosistemas altamente productivos que aportan diversos servicios ecosistémicos (p. ej. provisión y filtración de agua, protección de inundaciones y huracanes, fertilización y aporte de sedimentos, captura de carbono) (M.E.A., 2005; Costanza et al., 2008). Sin embargo, a nivel mundial son uno de los más amenazados debido a actividades humanas como la agricultura, la ganadería (Mitsch et al., 2009) y más recientemente la urbanización. México ha perdido $62 \%$ de sus humedales y específicamente los de la costa centro oeste del Golfo de México (estado de Veracruz) son de los más amenazados debido a la fuerte presión ganadera, ya que esta entidad ocupa el primer lugar a nivel nacional en producción de carne de bovino (Contreras-Espinosa y Warner, 2004; Landgrave y Moreno-Casasola, 2012; Moreno-Casasola et al., 2014).

Diversos estudios han asociado el pastoreo del ganado bovino con la pérdida de biodiversidad (Verdú et al., 2000; Watkinson y Ormerod, 2001; Durant et al., 2008) y con la disminución de la riqueza de especies nativas. Por ejemplo Dorrough et al. (2006) encontraron que en bosques con pastizales en Australia, la riqueza de plantas nativas está correlacionada negativamente con la intensidad agrícola y de pastoreo, mientras que varias especies exóticas se veían favorecidas por estas actividades. Sin embargo, existen estudios que mencionan que la riqueza de especies vegetales en ciertos ecosistemas puede verse beneficiada con el pastoreo (Dupré y Diekmann, 2001; Pykälä, 2005).

En algunos humedales del estado de California (EUA) y de Brasil se ha observado que zonas sin pastoreo presentan mayor cobertura de pastos exóticos y menor cobertura de especies nativas que áreas con pastoreo, y se ha hecho énfasis en que la ganadería no es necesariamente dañina para los humedales, siempre y cuando el pastoreo sea de baja intensidad (Marty, 2005; Junk y da Cunha, 2012). En México, el único trabajo que existe al respecto es el de Travieso-Bello et al. (2005), quienes reportaron que la ganadería puede disminuir la riqueza de especies nativas de humedales, y también la biomasa; sin embargo, en este estudio no se realizaron tratamientos excluyendo al ganado y solo se muestreó una época del año, por lo que no presentaron comparaciones temporales.

Es necesario empezar a generar conocimiento acerca del nivel de impacto que tiene la ganadería en diferentes tipos de humedales (manglares, selvas inundables, popales, tulares), principalmente en regiones con tendencia al aumento de esta actividad o en donde la ganadería ocupa grandes extensiones de estos ecosistemas. Esta información podría ayudar a mejorar las políticas de manejo ganadero, logrando una ganadería sustentable en la zona costera, y garantizando la conservación de las funciones y servicios ecosistémicos. En este trabajo se evaluó el efecto de la ganadería y la estacionalidad sobre la composición florística, cobertura vegetal, biomasa aérea (BA) y biomasa subterránea (BS) de los humedales herbáceos (popales) de la costa centro oeste del Golfo de México, específicamente los de la laguna de Alvarado, Veracruz, para responder las siguientes preguntas: 1) ¿La ganadería tiene un impacto negativo en estos humedales? y 2) ¿La variación estacional determina el nivel de impacto ganadero y la composición de la vegetación? La hipótesis de trabajo plantea que una densidad baja de ganado mantendrá la biodiversidad florística del humedal, presentándose cambios estacionales en la composición de las especies.

\section{Materiales y métodos}

\section{Área de estudio}

El área de estudio se localiza cerca de Alvarado, Veracruz, en la costa centro oeste del Golfo de México, entre los $18^{\circ} 46^{\prime} \mathrm{N}, 95^{\circ} 34^{\prime} \mathrm{E}$ y los $18^{\circ} 42^{\prime} \mathrm{N}, 95^{\circ} 58^{\prime} \mathrm{E}$ (Fig. 1). La altitud es de $10 \mathrm{~m}$ y el clima es cálido con abundantes lluvias en verano. La temperatura es de $22-26{ }^{\circ} \mathrm{C}$ y la media de la precipitación pluvial es de $1748.3 \mathrm{~mm}$ (INEGI, 2005). Los datos fueron obtenidos para el proyecto $\mathrm{N}^{\circ} 48247$ CONACYT-CONAGUA "Inventario, delimitación, caracterización y uso sustentable de los humedales de la cuenca del Río Papaloapan, México”. Otros datos derivaron de entrevistas con los propietarios de los terrenos, observaciones de campo y mediciones. 


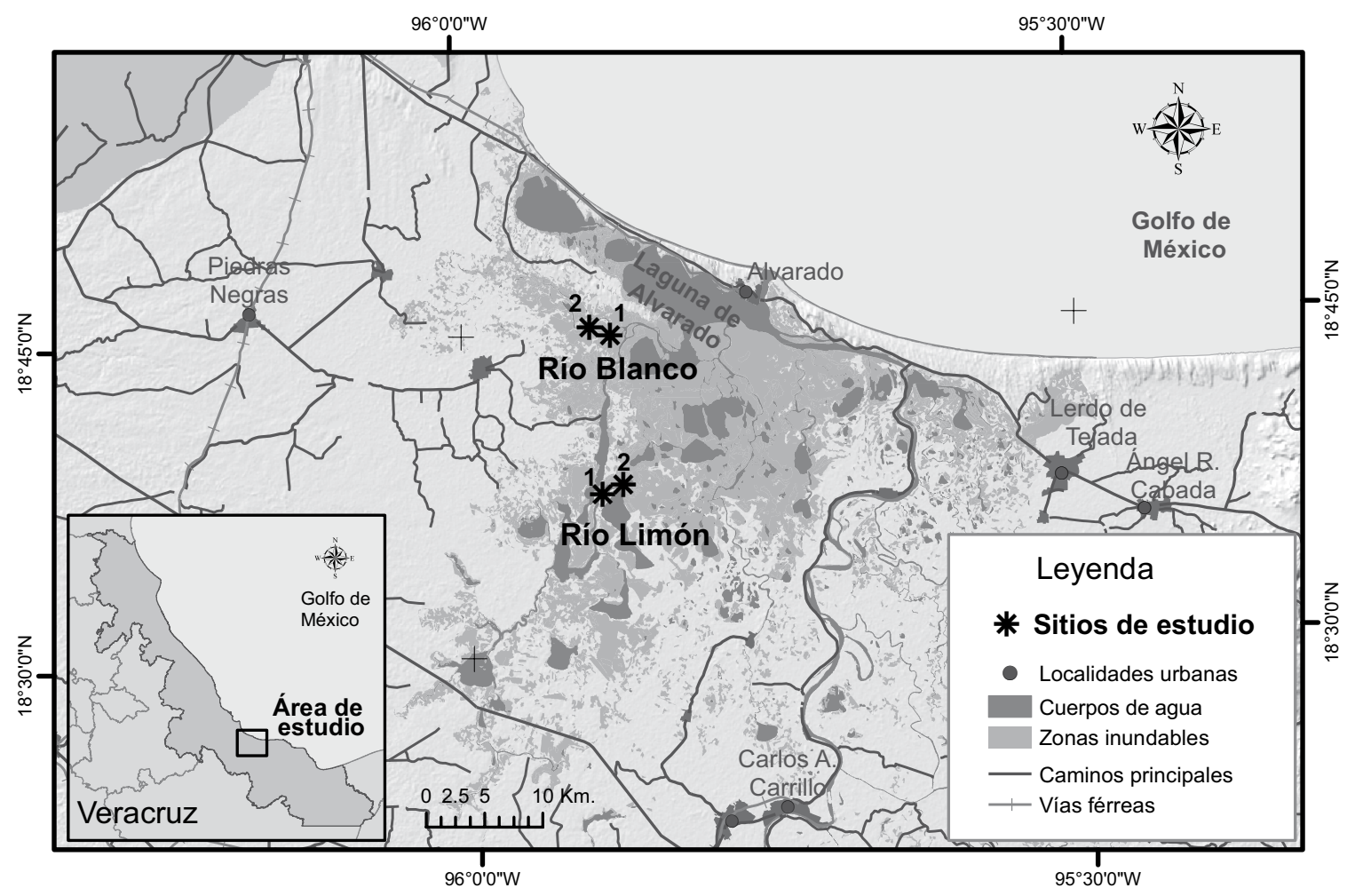

Figura 1: Localidades de Río Blanco (RB1, RB2) y Río Limón (RL1, RL2) en los humedales de Alvarado, Veracruz, México.

En los sitios de muestreo la vegetación natural que domina es el popal, término asignado por Faustino Miranda (Miranda y Hernández-X, 1963). Este ecosistema corresponde a una comunidad vegetal sobre superficies pantanosas permanentemente estancadas en la planicie costera de Tabasco, sur de Veracruz, Chiapas y Campeche. Se establece sobre llanuras aluviales, prácticamente sin declives, atravesadas por ríos que por medio de filtraciones e inundaciones cubren la superficie en zonas con climas tropicales (Rzedowski, 2006). La vegetación se caracteriza por presentar herbáceas emergentes enraizadas de 1 a $3 \mathrm{~m}$ de alto con hojas anchas. Algunas especies que se han reportado para los popales-tulares del estado de Veracruz son Typha domingensis Pers., Thalia geniculata L., Pontederia sagittata C. Presl, Sagittaria lancifolia L., Sagittaria latifolia Willd., Cyperus articulatus L., Cyperus giganteus Vahl, Eleocharis cellulosa Torr., Hymenachne amplexicaulis (Rudge) Nees, Heliconia latispatha Benth., Leersia ligularis Trin., Hydro- cotyle bonariensis Lam. y Bacopa monnieri (L.) Wettst. (Moreno-Casasola et al., 2010).

La economía del municipio de Alvarado está basada fundamentalmente en la ganadería bovina, en donde las razas más empleadas para esta actividad son el ganado Cebú para aprovechamiento de carne y el ganado Suizo y Holandés para aprovechamiento de leche (Portilla Ochoa, 2005). Es común que el pastoreo del ganado sea extensivo y también rotatorio, ya que se saca a los animales del terreno en el período de mayor inundación llevándolos a zonas más altas. La carga animal permitida en el municipio es de una cabeza de ganado por hectárea; sin embargo, en muchos ejidos esto no se respeta, excediendo la carga permitida (INEGI, 2007).

Para este estudio se seleccionaron cuatro sitios, todos impactados por la ganadería, ya que después de una búsqueda extensiva y conversaciones informales con los habitantes de la localidad y la Asociación Ganadera, no se encontraron humedales herbáceos totalmente libres de 
ganado para considerarlos como sitios control. Se seleccionaron dos localidades de trabajo, una fue Río Blanco, con dos sitios de muestreo (a partir de aquí llamados RB1 y RB2). La segunda localidad fue Río Limón, también con dos sitios de muestreo (RL1 y RL2). En RB1 el ganado es introducido aproximadamente en febrero o marzo y lo sacan aproximadamente en septiembre. En RB2 el ganado entra en enero y lo sacan en septiembre. En RL1 y RL2 el ganado es introducido entre enero y febrero y lo sacan entre octubre y noviembre. Ver Cuadro 1 para una descripción más detallada. Esta variación en el momento de extracción del ganado y el tiempo que permanece fuera del humedal depende del nivel de inundación de los predios particulares.

\section{Diseño experimental}

Debido a que no se encontró ningún popal no impactado por la ganadería, no se tuvo un sitio control. De tal mane- ra no se pudo medir la verdadera magnitud del impacto, $\mathrm{y}$ éste solo fue estimado de manera parcial, en relación con el sitio que presentó más características de un humedal herbáceo conservado.

RB1 y RB2 se encuentran separados entre sí por $2.5 \mathrm{~km}$, mientras que RL1 y RL2 por $2 \mathrm{~km}$. Los sitios de Río Blanco se encuentran a 14 km de los de Río Limón (Fig. 1). En cada sitio se colocaron ocho cuadros al azar de $1 \mathrm{~m}^{2}$ cada uno. Para evitar la entrada del ganado, cuatro de estos cuadros fueron cercados con alambre de púas de la siguiente manera: dos cuadros se colocaron dentro de un cerco de alambre de púas, cuidando que haya un espacio entre cuadros y el alambre exterior de al menos $1 \mathrm{~m}$, y en otra área del lugar se colocaron los otros dos cuadros de la misma manera. Se realizaron tres muestreos en temporada de secas (enero, marzo y mayo de 2009) y dos en la de inundación (septiembre y noviembre de 2009).

Cuadro 1: Características de los diferentes tipos de manejo en los cuatro sitios de muestreo localizados en el municipio de Alvarado, Veracruz, México.

\begin{tabular}{|c|c|c|c|c|}
\hline & RB1 & RB2 & RL1 & RL2 \\
\hline & $95^{\circ} 52^{\prime} 42.24^{\prime \prime O}$ & $93^{\circ} 53^{\prime} 36.38^{\prime \prime O}$ & $95^{\circ} 53^{\prime} 24.95^{\prime \prime O}$ & $95^{\circ} 52^{\prime} 30.87^{\prime \prime O}$ \\
\hline Manejo & 1 cabeza ha $^{-1}$ & 2 o 3 cabezas ha ${ }^{-1}$ & 2 o 3 cabezas ha ${ }^{-1}$ & 2 cabezas ha $^{-1}$ \\
\hline Influencia humana & $\begin{array}{l}\text { Muy poca, en los cinco } \\
\text { muestreos nunca se } \\
\text { observó a personas } \\
\text { trabajando en el terreno. }\end{array}$ & $\begin{array}{l}\text { Muy poca, en los } \\
\text { cinco muestreos solo } \\
\text { se observó dos veces } \\
\text { al capataz cuidando } \\
\text { el terreno. Solo iba de } \\
\text { entrada por salida. }\end{array}$ & $\begin{array}{l}\text { Se encuentra la casa del } \\
\text { dueño a } 120 \text { metros en } \\
\text { línea recta al oeste del } \\
\text { sitio. El dueño va entre } \\
\text { una y dos veces por día } \\
\text { al sitio en caballo para } \\
\text { arrear a las vacas. }\end{array}$ & $\begin{array}{c}\text { En el terreno se } \\
\text { encuentra la casa de los } \\
\text { dueños, el señor al menos } \\
\text { una vez al día arrea al } \\
\text { ganado en caballo. }\end{array}$ \\
\hline Humedad del suelo e inundación & $\begin{array}{l}\text { Permanece inundado } 9 \\
\text { meses/año, y el resto del } \\
\text { año el suelo se encuentra } \\
\text { saturado en agua. }\end{array}$ & $\begin{array}{l}\text { Permanece inundado } 8 \\
\text { meses/año; el suelo está } \\
\text { saturado en agua } 2 \text { meses } \\
\text { y seco el resto del año. }\end{array}$ & $\begin{array}{c}\text { Permanece inundado } 6 \\
\text { meses y seco el resto de } \\
\text { año. }\end{array}$ & $\begin{array}{l}\text { Permanece inundado } 9 \\
\text { meses/año, y el resto del } \\
\text { año el suelo se encuentra } \\
\quad \text { saturado en agua. }\end{array}$ \\
\hline Permanencia de ganado en el sitio & 6-10 meses & $8-10$ meses & 10 meses & 10 meses \\
\hline
\end{tabular}




\section{Estructura y composición florística}

En cada cuadro se tomaron datos de composición de especies vegetales, porcentaje de cobertura vegetal para cada especie por medio del método de cobertura-abundancia de Westhoff y Van der Maarel (1978). Se estimó la frecuencia y cobertura relativa, y con esto se obtuvo el Valor de Importancia Relativa (VIR) (Moreno-Casasola y López Rosas, 2009) para cada una de las especies. Para obtener los valores de diversidad y de equidad por sitio, temporalidad y tratamiento, se utilizó el índice de Shannon-Wiener y el índice de equidad de Pielou (Liang et al., 2007). El reemplazo de especies únicamente se realizó por sitio y se calculó por medio del índice de Whittaker (1972) para RB2, RL1 y RL2, en relación con el sitio RB1, ya que éste es el que recibe menos influencia humana y tiene menor carga animal.

La biomasa áerea (BA) se obtuvo cortando la vegetación al ras del suelo dentro de cada cuadro de muestreo. Se colectó toda la materia incluyendo la que estaba enraizada a ese nivel y la fitomasa seca y muerta. Esto fue al inicio (enero 2009) y al final del experimento (noviembre 2009), es decir en noviembre se colectó la biomasa que creció durante once meses en presencia o ausencia del ganado. Después de cada corte, el material se secó durante 120 horas a $65^{\circ} \mathrm{C}$ y posteriormente se pesó. La biomasa subterránea (BS) se obtuvo por medio de un núcleo de $15 \mathrm{~cm}$ de largo por $10 \mathrm{~cm}$ de diámetro. En cada esquina (por fuera) de cada cuadro de $1 \mathrm{~m}^{2}$ se tomaron cuatro muestras, durante los cinco muestreos. Estas muestras se lavaron en laboratorio hasta eliminar la mayor cantidad de tierra posible. Posteriormente se pusieron en flotación para obtener la mayor cantidad de material vivo (Cejudo y Capistrán, 2009). Para separar este material se utilizaron dos tamices, uno de $0.1 \mathrm{~mm}$ y otro de $0.039 \mathrm{~mm}$ de abertura para raíces finas. Una vez separadas las raíces, se secaron en un horno a $70{ }^{\circ} \mathrm{C}$ por 24 horas, para obtener el peso seco.

\section{Análisis estadísticos}

Para conocer si el índice de diversidad, la riqueza media de especies, la BA, la BS y el VIR por especie varían en- tre los sitios de estudio, se aplicó un ANOVA de una vía y la prueba de rangos múltiples de Tukey. Cuando las variables no pudieron ser normalizadas se utilizó una ANOVA por rangos de una vía de Kruskal-Wallis y la prueba de comparaciones múltiples de Dunn. La riqueza media por temporadas (secas-lluvias) se calculó tomando en cuenta los valores de cada uno de los ocho cuadros en cada sitio. La riqueza media, BA y BS entre temporadas (lluviassecas) y tratamientos (presencia de ganado y exclusión de ganado) se compararon mediante una prueba de $t$ pareada o si los datos no pudieron ser normalizados se utilizó una prueba de Rango de Wilcoxon (Zar, 1999). No se realizaron comparaciones estadísticas en los casos en donde la especie solo se presentaba en un solo tratamiento o una sola temporada, solo se menciona el porcentaje de VIR en donde la especie estuvo presente.

Para visualizar la similitud entre los sitios de muestreo y cuadros con y sin ganado en función de las variables medidas se realizaron análisis de componentes principales (ACP). Es importante mencionar que en los ACPs no se incluyeron todas las especies. Las especies que quedaron fuera del análisis son todas ruderales, a excepción de Lippia nodiflora (L.) Michx., y solo se presentaron en un sitio, en un muestreo (generalmente fue el de septiembre) y en un solo cuadro (de alguno de los ocho del sitio), por lo que fueron especies que no aportaron información relevante al análisis. El nivel de significancia utilizado en todos los análisis fue de 0.05 y se utilizaron los paquetes estadísticos R versión 2.5.1 (Crawley, 2014), Statgraphics plus 5.1 y MVSP 3.1. (Manugistics, 1998).

\section{Resultados}

\section{Variación por sitio}

Se registró un total de 29 especies (Anexo) y con base en los resultados obtenidos se encontró que había especies clave con VIRs arriba de 40 (Cuadros 2). Con respecto a la diversidad, riqueza, BA y BS, se observó un gradiente de perturbación entre los cuatro sitios, siendo RB1 el menos impactado por la ganadería, seguido de RB2, RL1 y RL2 (Cuadro 3). 
Cuadro 2: Se muestran únicamente las especies que presentaron los valores más altos de importancia relativa (VIR) para cada sitio de muestreo en la costa centro oeste del Golfo de México. El valor indica la media \pm 1 EE del VIR de los cinco muestreos del año. Se aplicó una ANOVA y la prueba de rangos múltiples de Tukey $\left(^{*}\right)$ solo en los casos en donde las especies fueron encontradas en más de dos sitios. Solo se encontraron diferencias significativas en Sagittaria lancifolia $\mathrm{L}$. $(\mathrm{F}=17.04, \mathrm{p}=0.00)$ que se encontró en los cuatro sitios, y en Thalia geniculata $\mathrm{L}$. (F=11.88, $\mathrm{p}=0.008$ ) presente en dos sitios. Valores con la misma letra son estadísticamente iguales con $p>0.05$.

\begin{tabular}{|c|c|c|c|}
\hline RB1 & & RB2 & \\
\hline Sagittaria lancifolia $\mathrm{L}$. & $77.3 \pm 2.79 * a$ & Echinochloa colona (L.) Link & $86.3 \pm 4.32$ \\
\hline Eleocharis cellulosa Torr. & $69.3 \pm 7.47$ & Eleocharis celulosa Torr. & $42 \pm 12.04$ \\
\hline Hydrocotyle verticillata Thunb. & $68.5 \pm 2.02$ & Bacopa monnieri (L.) Wettst. & $40.9 \pm 11.15$ \\
\hline Echinochloa colona (L.) Link & $68.5 \pm 17.57$ & Sagittaria lancifolia $\mathrm{L}$. & $21.7 \pm 6.3 * b$ \\
\hline Bacopa monnieri (L.) Wettst. & $56.1 \pm 5.84$ & Lemna minor $\mathrm{L}$. & $21.1 \pm 13.51$ \\
\hline \multirow[t]{2}{*}{ Sporobolus virginicus (L.) Kunth } & $25.7 \pm 16.77$ & Thalia geniculata $\mathrm{L}$. & $18.3 \pm 7.72 * a$ \\
\hline & & Nymphaea ampla (Salisb.) DC. & $16.5 \pm 10.13$ \\
\hline RL1 & & RL2 & \\
\hline Eleocharis cellulosa Torr. & $67.8 \pm 8.16$ & Hymenachne amplexicaulis (Rudge) Ness & $73.1 \pm 3.43$ \\
\hline Hymenachne amplexicaulis (Rudge) Ness & $66.1 \pm 17.38$ & Thalia geniculata $\mathrm{L}$. & $48.4 \pm 4.09 * b$ \\
\hline Leersia hexandra Sw. & $65.9 \pm 7.92$ & Ludwigia octovalvis (Jaq.) P.H. Raven & $54.2 \pm 2.34$ \\
\hline Sagittaria lancifolia $\mathrm{L}$. & $33.3 \pm 8.01 * b$ & Eleocharis cellulosa Torr. & $44.7 \pm 5.54$ \\
\hline Cyperus articulatus L. & $27.6 \pm 11.95$ & Pontederia sagittata C. Presl & $36.3 \pm 1.36$ \\
\hline \multirow[t]{3}{*}{ Nymphaea ampla (Salisb.) DC. } & $3.47 \pm 3.47$ & Ambrosia psilostachya DC. & $24.4 \pm 15.44$ \\
\hline & & Sagittaria lancifolia $\mathrm{L}$. & $24.0 \pm 6.84 * b$ \\
\hline & & Paspalum sp. & $26.3 \pm 16.17$ \\
\hline
\end{tabular}

Cuadro 3: Diversidad de especies (índice de Shannon $-H$ 'e índice de equidad de Pielou - $J$ '), riqueza de especies, biomasa aérea (BA) y biomasa subterránea (BS) para los cuatros sitios de muestreo en el municipio de Alvarado, Veracruz, México. En el caso del índice de Shannon se indica el valor de éste $\left(H^{\prime}\right)$ y el tamaño de muestra $(S)$. Se indica la media \pm 1 EE en los casos donde se aplicó un ANOVA y la prueba de rangos múltiples de Tukey (*) y se muestra la mediana y los percentiles (25-75\%) en los casos donde se aplicó un ANOVA por rangos de Kruskal-Wallis y una prueba de Dunn $(* *)$. Valores con la misma letra son estadísticamente iguales con $p>0.05$.

\begin{tabular}{|c|c|c|c|c|c|c|}
\hline Característica & RB1 & RB2 & RL1 & RL2 & Valor estadístico & Valor de $p$ \\
\hline Índice de diversidad & $S=9, H^{\prime}=1.81 \mathrm{~b}$ & $S=10, H^{\prime}=1.63 \mathrm{~b}$ & $S=9, H^{\prime}=1.6 \mathrm{~b}$ & $S=19, H^{\prime}=2.32 \mathrm{a}$ & $H=6.28$ & 0.04 \\
\hline Índice de equidad & $J^{\prime}=0.95 \mathrm{a}$ & $J^{\prime}=0.61 \mathrm{bc}$ & $J^{\prime}=0.85 \mathrm{a}$ & $J^{\prime}=0.77 \mathrm{ac}$ & $F=6.72$ & 0.00 \\
\hline Riqueza de especies* & $5 \pm 0.31 \mathrm{~b}$ & $5.6 \pm 1.36 \mathrm{~b}$ & $5.2 \pm 0.37 \mathrm{~b}$ & $9.2 \pm 1.28 \mathrm{a}$ & $F=4.71$ & 0.01 \\
\hline $\mathrm{BA}\left(\mathrm{g} \mathrm{m}^{-2}\right)^{*}$ & $660 \pm 106.2 \mathrm{a}$ & $352.9 \pm 86.7 \mathrm{~b}$ & $600 \pm 84.3 \mathrm{a}$ & $410.37 \pm 65.8 \mathrm{ab}$ & $F=2.87$ & 0.04 \\
\hline $\mathrm{BS}\left(\mathrm{g} \mathrm{m}^{-2}\right)^{* *}$ & $23.5(19.83-24.95) \mathrm{a}$ & $16.99(12.65-20) b$ & $13.91(9.72-17.25) b$ & $9.38(6.3-11.88) \mathrm{c}$ & $H=40.59$ & 0.00 \\
\hline $\mathrm{BA}: \mathrm{BS} * *$ & $24.62(19.11-32.62) \mathrm{ab}$ & $15.81(9.56-20.56) b$ & $39.4(27.31-54.84) \mathrm{a}$ & $43.11(18.55-59.8) a$ & $H=13.58$ & 0.00 \\
\hline
\end{tabular}


Los cuatro sitios compartieron solo dos especies: Sagittaria lancifolia y Eleocharis cellulosa, dos especies características de humedales. El VIR de la primera especie fue significativamente mayor en RB1 en comparación con los demás sitios, mientras que los valores para E. cellulosa no fueron significativamente diferentes entre sitios. Se encontraron otras especies compartidas en dos o tres sitios, aunque la única especie que presentó diferencias significativas en el VIR fue Thalia geniculata, en donde fue mayor el valor de éste en RL2 que en RB2 (Cuadro 2). Es importante mencionar que hay especies que solo se presentaron en un sitio y en un muestreo y por tanto sus VIR fueron muy bajos, por lo que no se presentan en el Cuadro 2 .

Los valores del índice de reemplazo de especies de Whittaker de cada sitio con respecto a RB1 fueron de 1.66 para RB2, 1.74 para RL1 y de 1.91 para RL2. Por tanto, RB2 es más parecido a RB1 y RL2 es el que más difiere de éste. En cuanto a la diversidad de especies, RL2 fue significativamente mayor con respecto a los de- más sitios, seguido de RB1, RB2 y RL1. De acuerdo al valor del índice de equidad de Pielou, el sitio RB1 fue en donde la proporción de la diversidad fue más equitativa, mientras que en RB2 y RL2, esta proporción no es homogénea y hay especies dominantes. En cuanto a la riqueza de especies, el sitio RL2 fue significativamente mayor al resto de los sitios (Cuadro 3). Los resultados de BA y BS por sitio serán descritos más adelante.

\section{Variación por temporalidad de secas-Iluvias y asociación con especies y sitios de muestreo} En el ACP se observa que los dos primeros componentes explicaron $46 \%$ de la varianza acumulada. El primero muestra un gradiente de intensidad de forrajeo y las variables de especies de mayor peso fueron Bacopa monnieri, Echinochloa colona (L.) Link e Hydrocotyle verticillata Thunb. (asociadas positivamente), así como Hymenachne amplexicaulis, Pontederia sagittata, Ludwigia octovalvis (Jacq.) P.H. Raven y Paspalum sp. (aso-

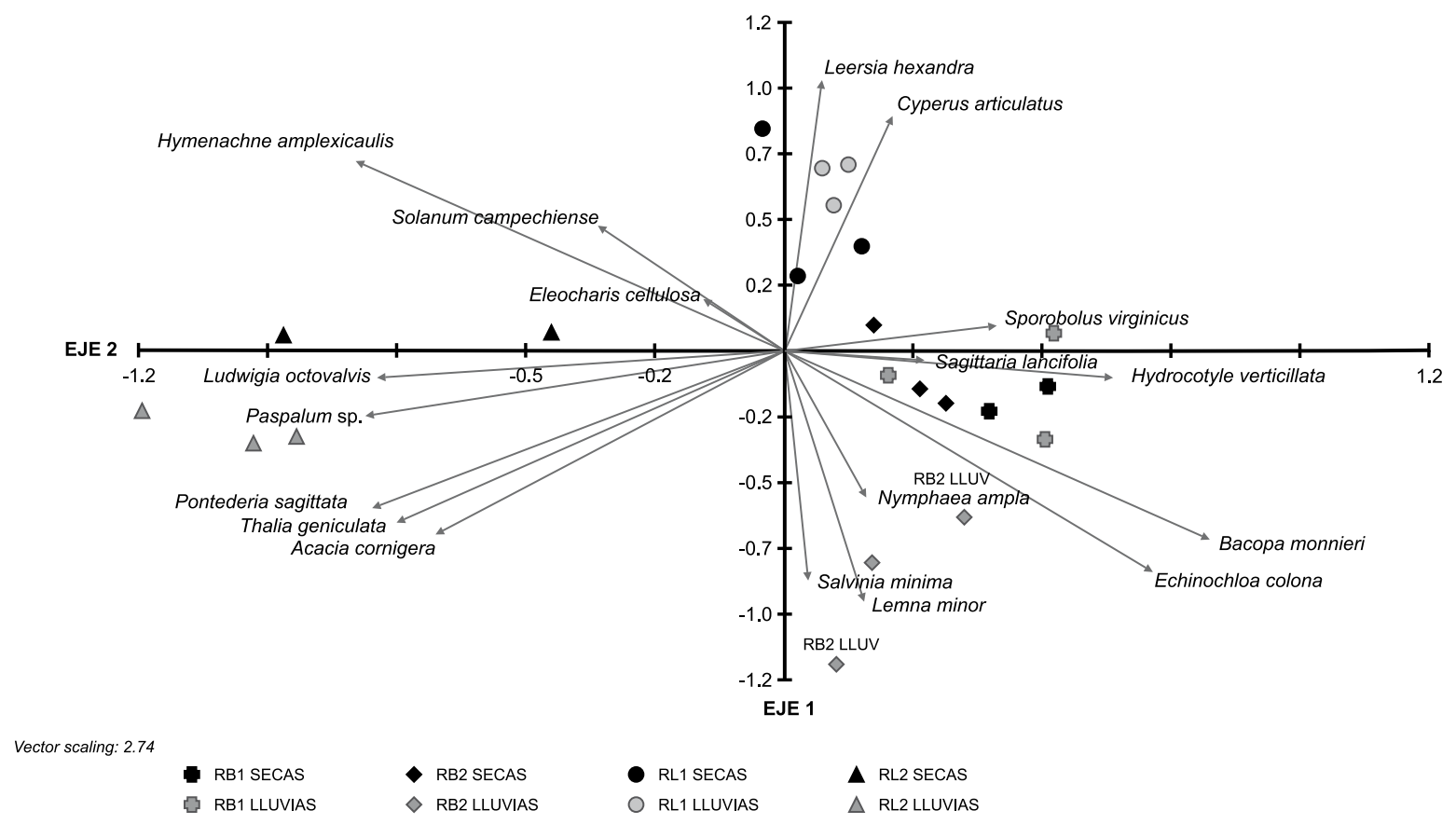

Figura 2: Asociación de las cuatro localidades de estudio (casos) con 18 especies (variables) con respecto a los dos componentes principales. Los dos componentes principales explican $46 \%$ de la variación. Sobre el primer componente se establece un gradiente de intensidad de forrajeo con los sitios de mayor intensidad en el extremo izquierdo (RL2). Dentro de cada sitio las épocas del año tienden a separarse sobre el eje dos en función de la temporalidad de secas-lluvias, excepto para el sitio RL1. 
ciadas negativamente). Esta última especie apareció solo en RL2 y en un muestreo. En el segundo el mayor peso correspondió a Cyperus articulatus asociada de manera positiva y Lemna minor L. y Nymphaea ampla (Salisb.) DC. asociadas negativamente (Fig. 2). Ambas especies solo aparecieron en la temporada de lluvias, y la primera únicamente en RB2.

Según el índice de Shannon, la diversidad de especies fue mayor en lluvias en todos los sitios, aunque las diferencias solo fueron significativas en RB2 (Secas $H^{\prime}=0.98$, Lluvias $H^{\prime}=1.83$ ) y en RL2 (Secas $H^{\prime}=1.8$, Lluvias $H^{\prime}=2.37$ ). El índice de equidad de Pielou reflejó que todos los sitios presentaron un mayor índice en lluvias; sin embargo, solo en RB1 y RB2 las diferencias fueron significativas. La riqueza media de especies fue mayor en lluvias en todos los sitios, aunque solo en RB1 las diferencias no fueron significativas (Cuadro 4).
Las especies que incrementaron su VIR y se vieron beneficiadas en los cuatro sitios durante la temporada de lluvias fueron Sagittaria lancifolia y Eleocharis cellulosa (Cuadro 5). En los sitios RB1 y RB2, la especie que incrementó su VIR en esta temporada fue Bacopa monnieri (RB1 Secas 47.6, Lluvias 68.92; RB2 Secas 30.55, Lluvias 56.36), al igual que lo hicieron Hymenachne amplexicaulis (Secas 52.31, Lluvias 86.9), Cyperus articulatus (Secas 11.34, Lluvias 52.08) en RL1, y Thalia geniculata (Secas 41.89, Lluvias 58.33) en RL2. Se encontraron especies que solo estuvieron presentes durante la temporada de lluvias como el pasto Sporobolus virginicus (L.) Kunth en RB1, y Lemna minor, Nymphaea ampla y Pontederia sagittata en RB2. Durante la temporada de secas la especie más importante en RB1 fue Echinochloa colona (Secas 90.27, Lluvias 35.87), mientras que en RB2 ésta misma se presentó constante durante todo el año

Cuadro 4: Diversidad de especies (índice de Shannon - $H^{\prime}$ ), riqueza de especies, biomasa aérea (BA) y biomasa subterránea (BS) por temporadas (Secas-Lluvias) en cada uno de los sitios de muestreo en el municipio de Alvarado, Veracruz, México. En el caso del índice de Shannon, se indica el valor de éste $\left(H^{\prime}\right)$ y la muestra (S). Se indica la media $\pm 1 \mathrm{EE}$ al aplicarse la prueba de $t$ pareada y se muestra la mediana y los percentiles $(25-75 \%)$ en los casos donde se aplicó la prueba de Rango de Wilcoxon. Valores con la misma letra son estadísticamente iguales con $p>0.05$.

\begin{tabular}{|c|c|c|c|c|c|c|}
\hline \multirow[b]{2}{*}{ Característica } & \multicolumn{2}{|c|}{ RB1 } & \multicolumn{4}{|c|}{ RB2 } \\
\hline & Secas & Lluvias & & Secas & Lluvias & \\
\hline Índice de diversidad & $\mathrm{S}=7, H^{\prime}=1.57 \mathrm{a}$ & $\mathrm{S}=7, H^{\prime}=1.81 \mathrm{a}$ & $z=0.88 p=0.37$ & $\mathrm{~S}=5, H^{\prime}=0.98 \mathrm{a}$ & $\mathrm{S}=10, H^{\prime}=1.83 \mathrm{~b}$ & $\mathrm{z}=2.67 \mathrm{p}=0.00$ \\
\hline Índice de equidad & $J^{\prime}=0.92 \mathrm{a}$ & $J^{\prime}=0.98 \mathrm{~b}$ & $z=2.53 \mathrm{p}=0.01$ & $J^{\prime}=0.68 \mathrm{a}$ & $J^{\prime}=0.98 \mathrm{~b}$ & $z=2.66 \mathrm{p}=0.00$ \\
\hline Riqueza de especies & $4.5(3.25-5) \mathrm{a}$ & $5(4-5) \mathrm{a}$ & $z=1.7 \mathrm{p}=0.08$ & $2(1-2) \mathrm{a}$ & $4(3-4) b$ & $z=3.32 \mathrm{p}=0.00$ \\
\hline $\mathrm{BA}\left(\mathrm{g} \mathrm{m}^{-2}\right)$ & $820 \pm 196 a$ & $500 \pm 48.3 \mathrm{a}$ & $t=1.52 \mathrm{p}=0.18$ & $185.37 \pm 62.7 \mathrm{a}$ & $520.5 \pm 142 b$ & $t=-2.39 \mathrm{p}=0.04$ \\
\hline $\mathrm{BS}\left(\mathrm{g} \mathrm{m}^{-2}\right)$ & $23.7 \pm 1.7 \mathrm{a}$ & $21.22 \pm 1.3 \mathrm{a}$ & $t=2.17 \mathrm{p}=0.06$ & $13.65 \pm 2.62 \mathrm{a}$ & $19.41 \pm 0.76 b$ & $t=-3.21 \mathrm{p}=0.01$ \\
\hline \multirow[t]{2}{*}{ BA:BS } & $34.06 \pm 7.55 \mathrm{a}$ & $24.13 \pm 3.34 \mathrm{a}$ & $t=1.19 \mathrm{p}=0.27$ & $15.21(0-18) a$ & $19.25(11.8-57.1) \mathrm{b}$ & $z=2,24 \mathrm{p}=0.02$ \\
\hline & \multicolumn{2}{|c|}{ RL1 } & \multicolumn{4}{|c|}{ RL2 } \\
\hline Característica & Secas & Lluvias & & Secas & Lluvias & \\
\hline Índice de diversidad & $\mathrm{S}=8, H^{\prime}=1.52 \mathrm{a}$ & $\mathrm{S}=6, H^{\prime}=1.54 \mathrm{a}$ & $z=1.01 \mathrm{p}=0,311$ & $\mathrm{~S}=10, H^{\prime}=1.8 \mathrm{a}$ & $\mathrm{S}=15, H^{\prime}=2.37 \mathrm{~b}$ & $\mathrm{z}=2.09 \mathrm{p}=0.03$ \\
\hline Índice de equidad & $J^{\prime}=0.89 \mathrm{a}$ & $J^{\prime}=0.99 \mathrm{a}$ & $z=1.19 \mathrm{p}=0.23$ & $J^{\prime}=0.78 \mathrm{a}$ & $J^{\prime}=0.97 \mathrm{a}$ & $z=1.75 \mathrm{p}=0.07$ \\
\hline Riqueza de especies & $2(2-2) a$ & $3(3-4) b$ & $z=3.03 \mathrm{p}=0.00$ & $2(2-3.75) \mathrm{a}$ & $3.5(3-4) b$ & $z=2.81 \mathrm{p}=0.00$ \\
\hline $\mathrm{BA}\left(\mathrm{g} \mathrm{m}^{-2}\right)$ & $730.7 \pm 108.3 a$ & $470.6 \pm 118 \mathrm{a}$ & $t=4.74 \mathrm{p}=0.00$ & $242.25 \pm 84.6 \mathrm{a}$ & $578 \pm 57.8 \mathrm{~b}$ & $t=-3.54 \mathrm{p}=0.00$ \\
\hline $\mathrm{BS}\left(\mathrm{g} \mathrm{m}^{-2}\right)$ & $14.78 \pm 1.43 \mathrm{a}$ & $13.46 \pm 1 \mathrm{a}$ & $t=0.55 \mathrm{p}=0.59$ & $7.82 \pm 1.16 \mathrm{a}$ & $9.77 \pm 1 \mathrm{a}$ & $t=-1.54 \mathrm{p}=0.16$ \\
\hline BA:BS & $54.09 \pm 8.64 \mathrm{a}$ & $35.21 \pm 7.38 \mathrm{a}$ & $t=2.23 \mathrm{p}=0.06$ & $29.49 \pm 9.96 \mathrm{a}$ & $62.92 \pm 11.51 \mathrm{~b}$ & $t=-2.41 \mathrm{p}=0.04$ \\
\hline
\end{tabular}


Cuadro 5: Valor de importancia relativa (VIR) de cada especie por temporalidad de secas-lluvias para los cuatro sitios de muestreo en el municipio de Alvarado, Veracruz, México. Se indica la media \pm 1 EE del Valor de Importancia Relativa. Las especies con asterisco (*) solo se presentaron en una temporada.

\begin{tabular}{lcclcc}
\hline & Secas & Lluvias & Secas & Lluvias \\
\hline Especie & \multicolumn{2}{c}{ RB1 } & Especie & RL1 \\
\hline Sagittaria lancifolia L. & $78.04 \pm 3.46$ & $76.28 \pm 6.34$ & Eleocharis cellulosa Torr. & $61.88 \pm 12.63$ & $76.6 \pm 7.62$ \\
Eleocharis cellulosa Torr. & $59.84 \pm 8.38$ & $83.53 \pm 3.27$ & Hymenachne amplexicaulis (Rudge) Ness & $52.31 \pm 27.7$ & $86.9 \pm 1.02$ \\
Hydrocotyle verticillata Thunb. & $65.83 \pm 1.71$ & $72.71 \pm 1.98$ & Sagittaria lancifolia L. & $21.06 \pm 1.85$ & $51.59 \pm 8.54$ \\
Bacopa monnieri (L.) Wettst. & $47.6 \pm 2.93$ & $68.92 \pm 5.56$ & Cyperus articulatus L. & $11.34 \pm 11$ & $52.08 \pm 6.9$ \\
Sporobolus virginicus (L.) Kunth* & 0 & $51 \pm 16.67$ & Leersia hexandra Sw. & $67.36 \pm 11.46$ & $63.64 \pm 15.02$ \\
Echinochloa colona (L.) Link & $90.27 \pm 3.02$ & $35.87 \pm 30$ & & & RL2 \\
\hline Especie & \multicolumn{2}{c}{ RB2 } & Especie & & \\
\hline Echinochloa colona (L.) Link & $85.09 \pm 7.39$ & $88.19 \pm 4.16$ & Hymenachne amplexicaulis (Rudge) Ness & $76.54 \pm 1.31$ & $68.05 \pm 8.33$ \\
Thalia geniculata L. & $19.21 \pm 10.06$ & $17.01 \pm 16$ & Ludwigia octovalvis (Jacq.) P.H. Raven & $50.15 \pm 0.9$ & $41.66 \pm 1.38$ \\
Eleocharis cellulosa Torr. & $27.54 \pm 14.4$ & $64 \pm 5.9$ & Thalia geniculata L. & $41.89 \pm 1.15$ & $58.33 \pm 1.38$ \\
Bacopa monnieri (L.) Wettst. & $30.55 \pm 15.36$ & $56.36 \pm 11.68$ & Eleocharis cellulosa Torr. & $38.88 \pm 4.86$ & $53.47 \pm 10.41$ \\
Sagittaria lancifolia L. & $13.42 \pm 6.9$ & $34.02 \pm 0.1$ & Sagittaria lancifolia L. & $20.83 \pm 11.47$ & $28.81 \pm 5.9$ \\
Lemna minor L. * & 0 & $52 \pm 12.5$ & Pontederia sagittata C. Presl & $35.87 \pm 1.85$ & $36.8 \pm 2.77$ \\
Nymphaea ampla (Salisb.) DC. * & 0 & $41.31 \pm 1.73$ & & \\
Pontederia sagittata C. Presl * & 0 & $31.25 \pm 8.33$ & & \\
\hline
\end{tabular}

con un VIR alto (Secas 85, Lluvias 88). El pasto Leersia hexandra Sw. en RL1 presentó un VIR alto durante todo el año (Secas 67.36, Lluvias 63.64), y en RL2 la gramínea Hymenachne amplexicaulis se encontró asociada a la temporada de secas con VIR más alto que en lluvias (Secas 76.54, Lluvias 68) (Cuadro 5).

\section{Variación entre tratamientos y asociación con las especies y sitios de muestreo}

El segundo ACP que se realizó para los tratamientos explicó $32 \%$ de la varianza entre los dos primeros componentes siendo éstos los más importantes. Las variables de mayor peso en el primer componente fueron las especies Bacopa monnieri, Echinochloa colona, Hydrocotyle verticillata y Sagittaria lancifolia (asociadas positivamente) y Ludwigia octovalvis, Hymenachne amplexicaulis y Paspalum sp. (asociadas negativamente). En el segundo componente los mayores pesos correspondieron a Thalia geniculata y Gomphrena hispida L., asociadas positivamente, e Hymenachne amplexicaulis y Eleocharis cellulosa asociadas negativamente (Fig. 3).

La riqueza media de especies fue significativamente mayor en el tratamiento "sin ganado" en todos los sitios excepto RB2. No se observaron diferencias en cuanto al Índice de diversidad de Shannon e Índice de equidad de Pielou entre los tratamientos (Cuadro 6). En el ACP se observa que las especies que se asocian al tratamiento "sin ganado" en el sitio RB1 fueron $H$. verticillata, $S$. lancifolia y B. monnieri, presentando diferencias significativas en cuanto al VIR solo las dos últimas especies mencionadas; en el sitio RB2 fueron B. monnieri y L. minor; en el sitio RL1 fueron L. hexandra, C. articulatus y E. cellulosa, presentando diferencias significativas en el VIR solo la primera especie; y en el sitio RL2 se observa 


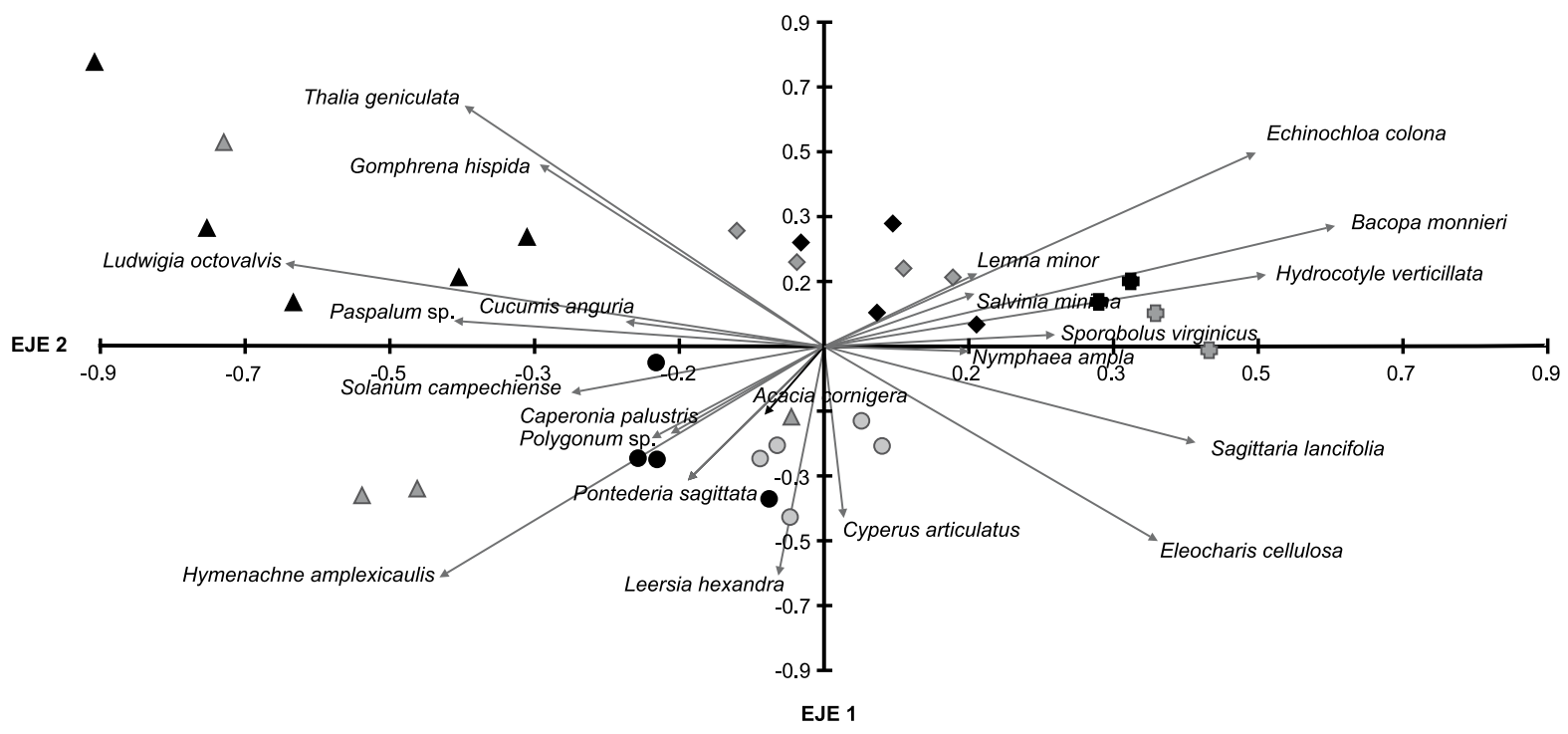

Vector scaling: 1.80

\begin{tabular}{|c|c|c|c|}
\hline RB1 CG & $\diamond \mathrm{RB} 2 \mathrm{CG}$ & - RL1 CG & $\Delta \mathrm{RL2} C \mathrm{G}$ \\
\hline RB1SG & $\diamond \mathrm{RB} 2 \mathrm{SG}$ & O RL1 SG & $\triangle R L 2 S G$ \\
\hline
\end{tabular}

Figura 3: Asociación de las cuatro localidades de estudio (casos) con respecto a los dos componentes principales. Dentro de cada localidad hay tratamientos "sin ganado" SG (color gris) y "con ganado" CG (color negro). Como variables se utilizaron 22 especies. Los dos componentes principales explican $32 \%$ de la variación.

la presencia exclusiva de $H$. amplexicaulis en este tratamiento. En cuanto al tratamiento "con ganado", en RB1 se observa como la gramínea $S$. virginicus es exclusiva de este tratamiento, mientras que en RB2 E. colona se asocia a la presencia del ganado presentando un VIR mayor, sin embargo, las diferencias no fueron significativas. En RL1, H. amplexicaulis se encuentra asociada a este tratamiento, y en RL2 las especies asociadas al ganado fueron especies como L. octovalvis, G, hispida, C. anguria y Paspalum sp. (Fig. 3, Cuadro 7).

\section{Biomasa por sitio, por temporalidad de secas- Iluvias y tratamiento}

En el sitio RB2 la BA $\left(352.9 \mathrm{~g} \mathrm{~m}^{-2}\right)$ fue significativamente menor que en RB1 $\left(660 \mathrm{~g} \mathrm{~m}^{-2}\right)$ y en RL1 $\left(600 \mathrm{~g} \mathrm{~m}^{-2}\right)$. Con respecto a la BS, en RB1 $\left(23.5 \mathrm{~g} \mathrm{~m}^{-2}\right)$ hubo mayor cantidad de ésta con respecto a los demás sitios y RL2 $\left(9.385 \mathrm{~g} \mathrm{~m}^{-2}\right)$ resultó menor estadísticamente a los sitios restantes (Cuadro 3). Con respecto a la relación BA:BS, RB2 (15.81) fue significativamente menor en comparación con RL1 (39.4) y RL2 (43.11). En cuanto a la temporalidad, en el sitio RB2 en lluvias la cantidad de BS, BA y la relación $\mathrm{BA}$ :BS fueron mayores. En RL2 solo BA y la relación $\mathrm{BA}: \mathrm{BS}$ fueron mayores estadísticamente en esta misma temporada (Cuadro 4). En los tratamientos "con ganado" y "sin ganado", no hubieron diferencias significativas en cuanto a la BA; sin embargo, en cuanto a la BS, ésta fue mayor en las parcelas "sin ganado" en RB1 (Cuadro 6).

\section{DISCUSIÓN}

La composición florística de los cuatro humedales herbáceos de Alvarado en la costa centro oeste del Golfo de México, estudiados en el presente trabajo, se está viendo afectada negativamente por la carga ganadera (dos cabezas de ganado por ha o más). Los resultados indican que el gradiente de transformación encontrado entre los sitios de muestreo se debe a las diferencias de manejo, tanto en carga animal como en el tiempo 
Cuadro 6: Diversidad y riqueza de especies, biomasa aérea (BA) y biomasa subterránea (BS) tomadas en las parcelas de cada uno de los sitios de estudio, de acuerdo al tratamiento ganadería ("sin ganado" y "con ganado") en el municipio de Alvarado, Veracruz, México. En el caso del índice de Shannon, se indica el valor de éste $\left(H^{\prime}\right)$ y la muestra (S). Se indica la media $\pm 1 \mathrm{EE}$ al aplicarse la prueba de $t$ pareada $(*)$ y se muestra la mediana y los percentiles (25-75\%) en los casos donde se aplicó la prueba de Rango de Wilcoxon (**). Valores con la misma letra son estadísticamente iguales con $p>0.05$.

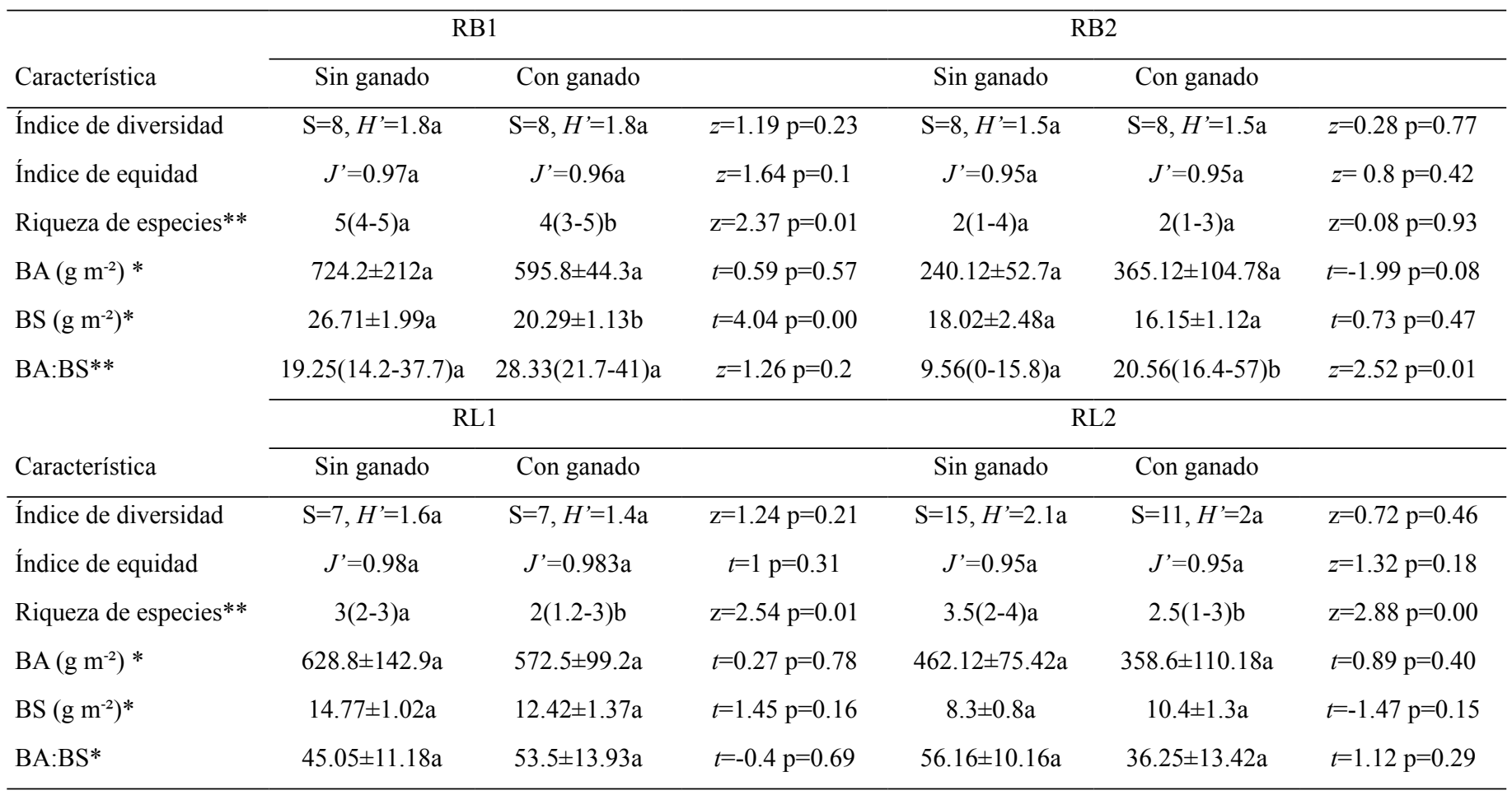

de permanencia en el predio, y esto a su vez causa alteración en la estructura vegetal. Se observa que RB1, sitio con menor carga animal, fue el más conservado debido a que las especies presentes son principalmente nativas del humedal estuvieron constantes a lo largo del año con altos VIRs (arriba de 50) y presentaron equidad en cuanto a la proporción de su abundancia según el índice de Pielou (0.95) (Cuadro 3). Las especies a las que nos referimos y que se han documentado como propias de humedales son Bacopa monnieri, Hydrocotyle verticillata, Sagittaria lancifolia y Eleocharis cellulosa (Moreno-Casasola et al., 2010). Estas últimas dos fueron las únicas especies compartidas en los cuatro sitios de muestreo, y principalmente Sagittaria lancifolia se encontró en mayor abundancia en las localidades menos perturbadas. Se ha reportado que esta especie es sensible a la presencia de algunas gramíneas exóticas como
Echinochloa pyramidalis (Lam.) Hitchc. \& Chase (López Rosas et al., 2010), la cual no se encuentra en nuestros sitios de muestreo, pero sí se reportó a Echinochloa colona que podría estar jugando el mismo papel, ya que está considerada como una especie exótica e invasora (Hanan y Mondragón, 2009). Durante la temporada de secas el índice de equidad de Pielou fue significativamente menor que en lluvias, lo que indica que hay una especie dominante la cual es Echinochloa colona según los resultados del VIR por temporalidad (Cuadro $4 \mathrm{y}$ 5). Sin embargo, el crecimiento de esta especie puede estar siendo controlado por la entrada de agua durante la temporada de lluvias y el régimen de inundación que permanece constante durante el año, y por tanto no se ve afectado el crecimiento de Sagittaria lancifolia. Esto coincide con los resultados del trabajo de López Rosas y Moreno-Casasola (2012), quienes encontraron que la 
Cuadro 7: Valor de importancia relativa (VIR) de las especies más representativas para los cuatro sitios de muestreo en el municipio de Alvarado, Veracruz, México. Estos valores se presentan para el tratamiento ganadería ("sin ganado" y "con ganado"). Se indica la media $\pm 1 E E$ al aplicarse la prueba de $t$ pareada y se muestra la mediana y los percentiles (25-75\%) en los casos donde se aplicó la prueba de rango de Wilcoxon. Valores con la misma letra son estadísticamente iguales con $\mathrm{p}>0.05$. Las especies que presentan asterisco $(*)$, solo aparecieron en uno de los tratamientos y no se pudo realizar prueba estadística.

\begin{tabular}{|c|c|c|c|}
\hline \multirow[b]{2}{*}{ Especie } & \multicolumn{2}{|c|}{ RB1 } & \multirow[b]{2}{*}{ Estadístico } \\
\hline & Sin ganado & Con ganado & \\
\hline Sagittaria lancifolia $\mathrm{L}$. & $83.33(81.9-83.3) \mathrm{a}$ & 63.42(63.42-81.9)b & $z=2.03, \mathrm{p}=0.04$ \\
\hline Eleocharis cellulosa Torr. & $76.85 \pm 7.3 \mathrm{a}$ & $60.83 \pm 9.31 \mathrm{a}$ & $t=2.5, \mathrm{p}=0.06$ \\
\hline Hydrocotyle verticillata Thunb. & $71.66 \pm 3.33 \mathrm{a}$ & $65.55 \pm 3.17 \mathrm{a}$ & $t=1.23, \mathrm{p}=0.28$ \\
\hline Echinochloa colona (L.) Link & $79.16(32-93) \mathrm{a}$ & $90.27(39-93) \mathrm{a}$ & $z=1.46, \mathrm{p}=0.14$ \\
\hline Bacopa monnieri (L.) Wettst. & $63.51 \pm 5.8 \mathrm{a}$ & $48.05 \pm 6.86 b$ & $t=3.47, \mathrm{p}=0.02$ \\
\hline \multirow[t]{2}{*}{ Sporobolus virginicus (L.) Kunth* } & 0 & $28 \pm 39.31$ & \\
\hline & \multicolumn{2}{|c|}{ RB2 } & \\
\hline Echinochloa colona (L.) Link & $80.55 \pm 7.77 \mathrm{a}$ & $91.94 \pm 1.83 \mathrm{a}$ & $t=-1.61, \mathrm{p}=0.18$ \\
\hline Eleocharis cellulosa Torr. & $39.72 \pm 17.7 \mathrm{a}$ & $31.94 \pm 13.5 \mathrm{a}$ & $t=0.41, \mathrm{p}=0.69$ \\
\hline Bacopa monnieri (L.) Wettst. & $61.11(0-72) \mathrm{a}$ & $0(0-54) \mathrm{a}$ & $z=1.09, \mathrm{p}=0.27$ \\
\hline Thalia geniculata $\mathrm{L}$. & $12.77 \pm 8.25 \mathrm{a}$ & $12.77 \pm 8.25 \mathrm{a}$ & $t=0, \mathrm{p}=1$ \\
\hline Pontederia sagittata C. Pres1* & $15 \pm 9.55$ & 0 & \\
\hline \multirow{2}{*}{ Lemna minor $\mathrm{L} . *$} & $20.5 \pm 12.59$ & 0 & \\
\hline & \multicolumn{2}{|c|}{ RL1 } & \\
\hline Eleocharis cellulosa Torr. & $67.91 \pm 6.42 \mathrm{a}$ & $45.27 \pm 18.69 \mathrm{a}$ & $t=1.2, \mathrm{p}=0.29$ \\
\hline Hymenachne amplexicaulis (Rudge) Ness & $47.5 \pm 20.02 \mathrm{a}$ & $67.22 \pm 17.81 \mathrm{a}$ & $t=-1.16, \mathrm{p}=0.31$ \\
\hline Leersia hexandra Sw. & $73.05 \pm 9.51 \mathrm{a}$ & $40 \pm 17.1 b$ & $t=3.45, \mathrm{p}=0.02$ \\
\hline Cyperus articulatus L. & $22.45 \pm 14.18 \mathrm{a}$ & $18.05 \pm 11.16 \mathrm{a}$ & $t=0.31, \mathrm{p}=0.76$ \\
\hline \multirow[t]{2}{*}{ Sagitaria lancifolia L.* } & $36.8 \pm 6.06$ & 0 & \\
\hline & \multicolumn{2}{|c|}{ RL2 } & \\
\hline Hymenachne amplexicaulis (Rudge) Ness & $93(81.25-97) \mathrm{a}$ & $0(0-59.7) b$ & $z=2.02, \mathrm{p}=0.04$ \\
\hline Ludwigia octovalvis (Jacq.) P.H. Raven & $52.77(0-55.5) \mathrm{a}$ & $56.94(51.3-67.36) \mathrm{a}$ & $z=1.46, \mathrm{p}=0.14$ \\
\hline Ambrosia psilostachya DC. & $23.33 \pm 14.52 \mathrm{a}$ & $26.29 \pm 16.73 a$ & $t=-1.27, \mathrm{p}=0.32$ \\
\hline Paspalum sp. & $23.33 \pm 14.31 \mathrm{a}$ & $29.44 \pm 18.16 \mathrm{a}$ & $t=-1.12, \mathrm{p}=0.32$ \\
\hline Pontederia sagittata C. Presl * & $42.5 \pm 1.36$ & 0 & \\
\hline Sagitaria lancifolia L.* & $30.27 \pm 8.5$ & 0 & \\
\hline Eleocharis cellulosa Torr.* & $51.11 \pm 4.5$ & 0 & \\
\hline
\end{tabular}

competencia entre Echinochloa pyramidalis y Sagittaria lancifolia era asimétrica en diferentes condiciones de inundación. Por ejemplo, en cuadrantes en donde el suelo se encontraba seco se veía favorecido el crecimiento de Echinochloa P. Beauv. y en cuadrantes en donde el suelo se mantenía inundado, el crecimiento de 
la gramínea era limitado y dominaba Sagittaria L.

El sitio RB2 fue el más conservado después de RB1. La mayoría de las especies de este sitio son propias de un humedal herbáceo; sin embargo, el pasto exótico Echinochloa colona fue el único que presentó un VIR alto durante todo el año (86\%) en comparación a todas las demás que no obtuvieron más de $42 \%$. Es probable que las condiciones de disturbio de este sitio (pisoteo del ganado y sequía) estén favoreciendo el crecimiento de esta especie (Mejía-Saulés y Aranda, 1992) lo que limita el crecimiento de las especies propias del humedal, que durante la época de lluvias claramente se ven favorecidas (López Rosas et al., 2010). Los sitios RL1 y RL2 son los que más difieren de un humedal herbáceo conservado. En ambos sitios la carga animal es mayor y además el ganado permanece por más tiempo. Esto podría tener como consecuencia una mayor compactación del suelo y modificaciones en la hidrología y por tanto se favorece el crecimiento de especies exóticas y se inhibe el de las especies nativas del humedal. Además, los sitios RB1 y RB2 contienen más materia orgánica debido al tipo de suelo, mientras que RL1 y RL2 carecen de ésta (Rodríguez-Medina y Moreno-Casasola, 2013).

Además del manejo ganadero, otro factor que influye en el nivel de impacto y en el cambio de la vegetación es la variación estacional. En dos de los cuatro sitios se observa mayor diversidad y en tres sitios mayor riqueza vegetal en lluvias (Cuadro 4), lo que sugiere que el régimen de inundación está determinando el crecimiento de especies propias del humedal (Hess et al., 2003). Sin embargo, algunos autores mencionan que, tanto en humedales como en otros ecosistemas, la riqueza de especies aumenta al inicio de la temporada de lluvias, debido al crecimiento de especies facultativas que aprovechan situaciones medias de disturbio, cambios en el hidroperíodo y nuevas condiciones de humedad. Esto podría estar sucediendo en RB2 y RL1, pero sobre todo en RL2, que fue el que presentó mayor riqueza de especies. En estos sitios, al inicio de la temporada de lluvias se registra un incremento en el número de especies no nativas del humedal; sin embargo, conforme avanza la temporada de lluvias, los niveles de inundación se elevan y la riqueza de especies disminuye debido a que muchas plantas ruderales no soportan las condiciones de inundación, y generalmente se mantienen especies que son tolerantes a estos ambientes, como son las hidrófitas (Pollock et al., 1998; Weiher, 2003; Travieso-Bello et al., 2005). En humedales perturbados en donde la hidrología ha sido modificada y el sitio permanece menor tiempo inundado (como el sitio RL2), la riqueza de especies nativas decrece e incrementa la de especies facultativas que suelen estar presentes durante todo el año, únicamente con variaciones en su abundancia (Grace y Pugesek, 1997; Travieso-Bello et al., 2005).

Algunas de las especies nativas de los humedales y otras introducidas desarrollan estrategias para tolerar las condiciones de inundación o sequía. En el presente trabajo es importante conocer algunas de estas adaptaciones para entender la permanencia o ausencia de las especies en los sitios. Por ejemplo, los estudios realizados por Busch et al. (2004) y Baksh y Richards (2006) mencionan que, bajo condiciones críticas de inundación, los brotes jóvenes de Eleocharis cellulosa al entrar en contacto con el agua crecen más rápido y producen tallos más gruesos y altos, generando una mayor biomasa aérea en relación con la biomasa subterránea. En secas, estos brotes mueren, pero la especie puede soportar largos períodos de estiaje y producir nuevos brotes, aunque con crecimiento más lento. El hecho de que Eleocharis cellulosa haya presentado un VIR bajo en todos los sitios en la temporada de secas comparado con lluvias puede deberse a lo mencionado anteriormente y también a que ésta es una especie de mayor palatabilidad para el ganado, el cual tiene mayor presencia en secas (Rosen et al., 2008). También se encontraron en los sitios tres especies de gramíneas dominantes: Hymenachne amplexicaulis, Leersia hexandra y Echinochloa colona. La primera es una especie nativa pero invasora (especie translocada) (Hill, 1996; Houston y Duivenvoorden, 2003; Gordon et al., 2007) y se utiliza como forrajera de alta calidad. Su éxito reproductivo se basa en que es estolonífera con culmos que se dispersan sobre el suelo húmedo o flotan en la superficie del agua, a partir de los cuales producen raíces y forman densas 
colonias (Mejía-Saulés y Aranda, 1992), requiriendo de la temporada de secas para establecerse (Hill, 1996). Los sitios RL1 y RL2 presentan ambos escenarios, y en secas la planta logra establecerse ya que el sitio no se encuentra saturado en agua debido a la compactación del suelo originada por la ganadería, mientras que en lluvias el sitio se inunda logrando que la planta se disperse y se reproduzca con éxito. Esto explica por qué Hymenachne amplexicaulis fue tan exitosa en RL2 y RL1. En este último sitio, además de esta especie también se presentó Leersia hexandra, especie nativa pero invasora, que además es un pasto forrajero de primera calidad utilizado en el pastoreo, muy común en zonas inundables y puede tolerar hasta un metro de inundación (Mejía-Saulés y Aranda, 1992). Esta especie presentó un VIR alto durante todo el año, lo que sugiere que es muy plástica, con capacidad de adaptación a la sequía e inundación.

La tercera gramínea presente en los sitios fue Echinochloa colona, un pasto exótico que habita en lugares húmedos de regiones tropicales y subtropicales, y que a diferencia de Hymenachne amplexicaulis solo tolera parcialmente las inundaciones. Prefiere la temporada de estiaje y tiene la capacidad de adaptarse a suelos pobres en nutrientes (Mejía-Saulés y Aranda, 1992; Calderón y Rzedowski, 2004). Esta especie estuvo presente en RB1 y RB2 siendo más importante en el segundo sitio, en donde no se presentaron diferencias en el VIR entre secas y lluvias. Esto se puede deber a que es un sitio que no está inundado o saturado todo el año y además la carga animal es elevada, lo que se traduce en mayor pisoteo del ganado y mayor compactación. Esto podría indicar que es un suelo pobre en nutrientes, lo cual favorece el establecimiento de Echinochloa colona durante todo el año. A diferencia de RB2, en RB1 el VIR de esta especie fue mucho más alto en secas que en lluvias, lo que coincide con lo mencionado anteriormente por Mejía-Saulés y Aranda (1992) de que tolera parcialmente las inundaciones y prefiere la época de estiaje.

Se ha reportado que en los humedales de agua dulce del sureste de México la introducción de gramíneas y las prácticas ganaderas han causado pérdida de la biodi- versidad, ya que muchas de las especies nativas son reemplazadas por las introducidas y las prácticas ganaderas no les permiten mantenerse en el sitio (Travieso-Bello et al., 2005; López Rosas y Moreno-Casasola, 2012). De acuerdo a nuestros resultados esta situación puede estar presentándose en RB2, RL1 y RL2, ya que la ganadería ha favorecido que las tres gramíneas antes mencionadas (una de ellas exótica) dominen sobre las especies propias de los humedales.

\section{Variación por tratamiento ("con ganado" y "sin ganado")}

Se encontraron diferencias significativas entre sitios en cuanto a la carga animal, resultando ser RB1 (sitio más conservado y con poca carga animal), el sitio que presentó mayores características propias a las de un humedal conservado. Sin embargo, en cuanto al tratamiento las diferencias solo fueron significativas en la riqueza de especies y en BS en RB1. Por ejemplo, en las localidades de Río Limón en donde la riqueza fue significativamente mayor al excluir al ganado, se puede observar que las especies que se vieron favorecidas fueron básicamente las gramíneas y algunas ruderales como Leersia hexandra, Hymenachne amplexicaulis, Ambrosia psilostachya DC. y Paspalum sp., esto se debe a que, en áreas muy impactados por esta actividad, los humedales han perdido características intrínsecas del ecosistema, sobre todo en el componente suelo que es primordial para que la composición florística y la hidrología se mantengan. Las características del suelo que son afectadas por la ganadería son la capacidad de retención de agua, el espacio aéreo (porosidad), cantidad de materia orgánica y de micro y macro nutrientes (Rodríguez-Medina y Moreno-Casasola, 2013). Estas alteraciones traen como consecuencia que, aunque se excluya al ganado de la zona, las condiciones inicialmente son más favorables para especies dominantes como son las gramíneas y ruderales, limitando el crecimiento de la vegetación propia del humedal (Bantilan-Smith et al., 2009).

\section{BIOMASA}

El hecho de que la cantidad de BA en RB1 fuera estadís- 
ticamente mayor en comparación con los otros sitios se debe a que muchas especies propias de humedales herbáceos, al verse sometidas a situaciones de anaerobiosis por inundación constante (sitio RB1 casi inundado todo el año), aumentan su tamaño (por ejemplo, tallos más anchos y altos, y/u hojas más anchas), con lo que generan más aerénquima y pueden captar más oxígeno y transportarlo hacia las raíces (Moreno-Casasola y Wagner, 2009; Mitsch et al., 2009). Por ejemplo, López-Rosas y MorenoCasasola (2012) encontraron que especies propias del humedal, como Sagittaria lancifolia y Typha domingensis, generaban una cantidad estadísticamente mayor de biomasa en condiciones de inundación que durante la temporada de secas, y observaron lo contrario con el pasto invasor Echinochloa pyramidalis. En el presente trabajo no se midió la biomasa por especie; sin embargo, en RB1 la especie de mayor VIR fue Sagittaria lancifolia (probablemente debido a las condiciones de inundación), lo que podría explicar las altas cantidades de BA (Cuadro 2).

En el caso de RL1 y RL2, donde también se obtuvieron grandes cantidades de BA, las especies nativas de los humedales no fueron dominantes, lo que se explica por la variación del hidroperíodo y a la elevada carga animal. Sin embargo, en estas localidades el pasto Hymenachne amplexicaulis fue abundante y se ha reportado que esta especie genera gran cantidad de biomasa aérea (Gordon y Feo, 2007), al igual que Leersia hexandra, gramínea que también estuvo presente en RL1 (Calderón y Rzedowski, 2004).

En cuanto a los tratamientos, se observa que en RB1, RL1 y RL2, la cantidad de BA fue mayor en el tratamiento "sin ganado"; sin embargo, las diferencias no son significativas estadísticamente. El incremento de BA en ausencia de ganado se debe a que la ausencia de herbivoría acumula mayor biomasa vegetal seca y en descomposición, y a que la fitomasa viva no es consumida por los herbívoros (Olff y Ritchie, 1998; Travieso-Bello et al., 2005; Menghi et al., 2011). Es importante mencionar que en el primer muestreo del tratamiento "sin ganado" del sitio RB2 hubo vandalismo y se robó el alambre de púas que rodeaba los cuadros, permitiendo el acceso de ganado al tratamiento y la presencia de herbivoría. Esto hecho pudo influir en la cantidad de BA encontrada en este sitio $\mathrm{y}$ en los resultados entre tratamientos. Otro aspecto importante a considerar es que la BA que se obtuvo al inicio del experimento fue cortada dentro de los cuadros a muestrear, y esto pudo tener un efecto inicial de herbívora, e influir en los resultados entre tratamientos. Sin embargo, en cuanto a temporadas (lluvias-secas) no afectó ya que sí se observaron diferencias estadísticamente significativas. La biomasa subterránea fue significativamente mayor en RB1 (sitio permanentemente saturado o inundado). Esto es común que suceda en humedales herbáceos en buen estado de conservación, ya que cuando algunas plantas de estos ecosistemas se encuentran en situaciones de anaerobiosis, la disponibilidad de nutrientes es limitada, estimulando que las plantas generen mayor cantidad de biomasa en las raíces, aumentando así su área y la capacidad de absorción (Ponnamperuma, 1972; Chapin, 1980; Trought y Drew, 1980). Además, en condiciones de anaerobiosis la descomposición de la materia orgánica es lenta, generando un incremento en la acumulación de raíces muertas en proceso de descomposición en el suelo (Richardson y Vepraskas, 2001). El hecho de que RL1 y RL2 fueran los sitios con menor cantidad de BS posiblemente se deba a que son sitios con mucha carga animal y por consecuencia con suelos compactados, por lo que las raíces tienen limitado su crecimiento (Unger y Kaspar, 1994) (Cuadro 3).

Como ya se mencionó anteriormente, la ganadería es una actividad que va en aumento debido a la demanda productiva que existe, al aumento del precio de la carne de res y a las necesidades económicas de los pobladores, lo que se ve reflejado en un incremento de terrenos destinados a esta actividad. Ecosistemas como los humedales que antes no se veían afectados, ahora lo están. Esta actividad de manera intensiva, además de perturbar negativamente la composición florística, afecta otros componentes sumamente importantes para el buen funcionamiento de los humedales, como son las propiedades fisicoquímicas del suelo y la hidrología (Rodríguez-Medina y MorenoCasasola, 2013) y por tanto también se afectan servicios ecosistémicos dependientes de la porosidad del suelo 
como son el almacenamiento de carbono y agua en este componente (Campos et al., 2011). Sin embargo, existen estudios que mencionan que en diversos ecosistemas, incluyendo los humedales, la ganadería moderada, específicamente la herbivoría, funciona como control de especies dominantes (principalmente gramíneas) y favorece el crecimiento de las especies nativas. Con los resultados observados en este trabajo, coincidimos con esta idea de que los humedales herbáceos de agua dulce pueden conservar sus funciones ecológicas en presencia de la ganadería, siempre y cuando ésta sea de bajo impacto. Además, creemos que esta actividad de manera controlada puede favorecer a la diversidad de especies nativas de los humedales y limitar el crecimiento de los pastos forrajeros introducidos (Marty, 2005; Junk y da Cunha, 2012; Junk et al., 2014). Un ejemplo de esto es el humedal del sitio RB1. La idea sería moderar dos aspectos: 1) el tiempo en el que el ganado se encuentra en los humedales y 2) la carga animal. Podría considerarse también rotar el ganado más de una vez al año con el objetivo de dejar "descansar" el terreno y dar oportunidad a que la vegetación nativa se recupere. Otra práctica de manejo que se podría combinar con la ganadería de bajo impacto son pequeñas quemas controladas de bajas temperaturas (asegurando que no hay fuerte acumulación de material seco), ya que se ha reportado que se utilizan para frenar la reproducción vegetativa de especies invasoras (López Rosas et al., 2010). Sin embargo, habría que profundizar más en esta área y evaluar qué tanto estas pequeñas quemas podrían contribuir más a la recuperación del humedal o en su defecto perjudicarlo. En municipios como Tecolutla, Veracruz, se ha reportado de manera informal por los pobladores, que anualmente hay fuegos espontáneos en tulares, que son atendidos por los habitantes o autoridades correspondientes para evitar se propaguen a cultivos o caseríos, y que estas quemas no han afectado hasta el momento la presencia de las especies dominantes del humedal.

Concluimos de acuerdo a nuestros resultados obtenidos que: 1) la ganadería está teniendo un efecto negativo en tres de los humedales herbáceos muestreados y esto está relacionado con la carga animal y el tiempo de inundación.
Con respecto a los tratamientos "con y sin ganado" por sitio en general no se observaron diferencias estadísticamente significativas; 2) La variación estacional está determinando la composición florística de cada sitio e influyendo en el nivel de impacto ganadero. Por tanto, retomamos la hipótesis y consideramos que una ganadería con una carga de una cabeza por hectárea permite conservar la composición de especies nativas del humedal y un crecimiento en biomasa aérea y subterránea de esta vegetación comparable a la de un humedal sin impacto ganadero. Los autores están conscientes de las limitaciones que el diseño experimental tiene para los resultados, por lo que se sugiere que para futuros estudios el tiempo de muestreo se extienda a dos o más años, para que las diferencias entre tratamientos con respecto a la composición florística y la biomasa sean más evidentes. También se recomienda que los cuadros cercados para excluir al ganado sean de mayor tamaño que 1 $\mathrm{m}^{2}$, ya que con parcelas tan pequeñas hubo una fuerte presión de la ganadería externa. Cabe decir que este diseño podrá implementarse gracias a la información obtenida en el presente estudio. El hecho de no poder encontrar parcelas sin ganado, y que haya sido complicado encontrar parcelas sin menos de dos cabezas de ganado por ha, aun cuando en muchas zonas rurales del país con vegetación terrestre hay abandono de terrenos por ser improductivos, muestra la presión que está ejerciendo la ganadería en estos humedales. En la zona, a pesar de ser un sitio Ramsar, no hay Áreas Naturales Protegidas ni estaciones de campo donde se puedan montar experimentos a más largo plazo. Los resultados obtenidos pueden ser la base para convencer a algunos ganaderos locales de permitir usar una mayor superficie de su terreno por varios años, y contar con su apoyo para la protección de materiales asegurando que se impida la entrada del ganado.

Estos resultados, junto con los obtenidos en Brasil, son los primeros que se enfocan a obtener información sobre las implicaciones de la actividad ganadera en los humedales tropicales, una actividad ampliamente difundida en todos los continentes. Nuestros datos indican la necesidad de llevar a cabo experimentos más extensos, en distintos tipos de humedales y bajo diversos tipos de 
manejo, para poder dar recomendaciones que aseguren la sustentabilidad del funcionamiento del humedal, así como de una actividad económica fundamental para las zonas rurales del trópico húmedo.

\section{CONTRIBUCIONES DE AUTORES}

PMC y KRM concibieron y diseñaron el estudio. KRM y CYA realizaron los muestreos. KRM realizó los análisis. KRM y PMC contribuyeron a la interpretación de los datos. KRM escribió el manuscrito con la revisión y crítica de PMC y CYA. Todos los autores contribuyeron a la discusión, revisión y aprobación del manuscrito final.

\section{FINANCIMIENTO}

Este trabajo fue apoyado con recursos del proyecto $\mathrm{N}^{\circ}$ 48247 CONACYT-CONAGUA (Consejo Nacional de Tecnología y Comisión Nacional del Agua), proyecto $\mathrm{N}^{\circ}$ 107754 CONACYT-SEMARNAT (Consejo Nacional de Tecnología y Secretaria de Medio Ambiente y Recursos Naturales), y el Instituto de Ecología, A.C. Agradecemos al Consejo Nacional de Ciencia y Tecnología por la beca de maestría otorgada al primer autor (CONACyT CVU 275430/Reg. becario 224618).

\section{AgRADECIMIENTOS}

Agradecemos a Joaquín Cano y Gildardo Palacios por permitirnos instalar nuestros experimentos en sus terrenos, a Tiolino y Alberto Azua por la colaboración en el trabajo de campo, a Claudia Gallardo Hernández por la identificación de las gramíneas, y a Roberto Monroy Ibarra por la edición de las imágenes.

\section{LITERATURA CITADA}

Baksh, S. I. y J. H. Richards. 2006. An architectural model for Eleocharis: morphology and development of Eleocharis cellulosa (Cyperaceae). American Journal of Botany 93(5): 707-715. DOI: http://dx.doi.org/10.3732/ ajb.93.5.707

Bantilan-Smith, M., G. L. Bruland, R. A MacKenzie, A. R. Henry y C. R. Ryder. 2009. A comparison of the vegetation and soils of natural, restored, and created coastal lowland wetlands in Hawai' $i$. Wetlands 29(3): 1023-1035. DOI: http://dx.doi.org/10.1672/08-127.1

Busch, J., I. A. Mendelssohn, B. Lorenzen, H. Brix y S. L. Miao. 2004. Growth responses of the Everglades wet prairie species Eleocharis cellulosa and Rhynchospora tracyi to water level and phosphate availability. Aquatic Botany 78(1): 37-54. DOI: http://dx.doi.org/10.1016/j. aquabot.2003.09.002

Calderón, G. y J. Rzedowski. 2004. Manual de malezas de la región de Salvatierra, Guanajuato. Flora del Bajío y de Regiones Adyacentes XX: 1-315.

Campos Cascaredo, A., M. E. Hernández, P. Moreno-Casasola, E. Cejudo Espinosa, A. Robledo Ruiz y D. Infante Mata. 2011. Soil water retention and carbon pools in tropical forested wetlands and marshes of the Gulf of Mexico. Hydrological Sciences Journal 56(8): 1-19. DOI: http:// dx.doi.org/10.1080/02626667.2011.629786

Cejudo, E y A. Capistrán. 2009. Metodología para medir la productividad subterránea. In: Moreno-Casasola, P. y B. Warner (eds.). Breviario para describir, observar y manejar humedales. Serie Costa Sustentable no 1. RAMSAR, Instituto de Ecología, A.C., Comisión Nacional de Áreas Naturales Protegidas (CONANP), US Fish and Wildlife Service, US State Department. Xalapa, México. Pp. 265268.

Chapin, F. S. 1980. The mineral nutrition of wild plants. Annual Review of Ecology and Systematics 11: 233-260. DOI: http://dx.doi.org/10.1146/annurev.es.11.110180.001313

Contreras-Espinosa, F. y B. G. Warner. 2004. Ecosystem characteristics and management considerations for coastal wetlands in Mexico. Hydrobiologia 511(1): 233-245. DOI: http://dx.doi.org/10.1023/B:HYDR.0000014097.74263.54

Costanza, R., O. Pérez-Maqueo, M. L. Martínez, P. Sutton, S. J. Anderson y K. Mulder. 2008. The value of coastal wetlands for hurricane protection. AMBIO: A Journal of the Human Environment 37(4): 241-248. DOI: http://dx.doi. org/10.1579/0044-7447(2008)37[241:TVOCWF]2.0. $\mathrm{CO} ; 2$

Crawley, M. J. 2014. Statistics: an introduction using R. John Wiley and Sons. London, UK. 
Dorrough, J., C. Moxham, V. Turner y G. Sutter. 2006. Soil phosphorus and tree cover modify the effects of livestock grazing on plant species richness in Australian grassy woodland. Biology Conservation 130(3): 394-405. DOI: http://dx.doi.org/10.1016/j.biocon.2005.12.032

Dupré, C. y M. Diekmann. 2001. Differences in species richness and lifehistory traits between grazed and abandoned grasslands in southern Sweden. Ecography 24(3): 275286. DOI: http://dx.doi.org/10.1111/j.1600-0587.2001. tb00200.x

Durant, D., M. Tichit, E. Kerneis y H. Fritz. 2008. Management of agricultural wet grasslands for breeding waders: integrating ecological and livestock system perspectives-a review. Biodiversity Conservation 17(9): 2275-2295. DOI: http://dx.doi.org/10.1007/s10531-007-9310-3

Gordon, E. y Y. Feo. 2007. Dinámica de crecimiento de $H y$ menachne amplexicaulis en un humedal herbáceo en el Estado Miranda (Venezuela). Growth dynamics of Hymenachne amplexicaulis in a herbaceous wetland in Miranda state (Venezuela). Acta Botánica Venezuelica 30(1): 1-18.

Gordon, E., Y. Feo y L. Suárez. 2007. Efecto de la profundidad del agua sobre el crecimiento y biomasa de Hymenachne amplexicaulis en un humedal (estado Miranda, Venezuela). Revista de la Facultad de Agronomía de La Universidad del Zulia 24(1): 214.

Grace, J. B. y B. H. Pugesek. 1997. A structural equation model of plant species richness and its application to a coastal wetland. American Naturalist 149(3): 436-460.

Hanan, A. M y J. Mondragón. 2009. Malezas de México. Ficha Echinochloa colona. http://www.conabio.gob.mx/ malezasdemexico/poaceae/echinochloa-colona/fichas/ficha.htm (consultado agosto de 2016).

Hess, L. L., J. M. Melack, E. M. Novo, C. C. Barbosa y M. Gastil. 2003. Dual-season mapping of wetland inundation and vegetation for the central Amazon basin. Remote Sensing of Environment 87(4): 404-428. DOI: http://dx. doi.org/10.1016/j.rse.2003.04.001

Hill, K. U. 1996. Hymenachne amplexicaulis: a review of the literature and summary of work in Florida. University of Florida Extension. http://www.naples.net/ kuh/hymen. htm (consultado octubre de 2016).

Houston, W. A. y L. J. Duivenvoorden. 2003. Replacement of littoral native vegetation with the ponded pasture grass Hymenachne amplexicaulis: effects on plant, macroinvertebrate and fish biodiversity of backwaters in the Fitzroy River, Central Queensland, Australia. Marine and Freshwater Research 53(8): 1235-1244. DOI: http://dx.doi. org/10.1071/MF01042

INEGI. 2005. Principales resultados por localidad 2005 (ITER). Instituto Nacional de Estadística y Geografía. México, D.F. México. http://www.inegi.org.mx/sistemas/consulta_resultados/iter2005.aspx?c=27436\&s=est (consultado septiembre de 2009).

INEGI. 2007. Censo ejidal Veracruz. Total de ejidos y comunidades según tipo de actividad agropecuaria o forestal. Instituto Nacional de Estadística, Geografía e Informática. México, D.F., México. http://www.inegi.org.mx/est/ contenidos/proyectos/Agro/ca2007/Resultados_Ejidal/ default.aspx (consultado noviembre de 2010).

Junk, W. J. y C. N. da Cunha. 2012. Pasture clearing from invasive woody plants in the Pantanal: a tool for sustainable management or environmental destruction? Wetland Ecoloy and Managemet 20(2): 111-122. DOI: http://dx. doi.org/10.1007/s11273-011-9246-y

Junk, W. J., M. T. F. Piedade, R. Lourival, F. Wittmann, P. Kandus, L. D. Lacerda y A. A. Agostinho. 2014. Brazilian wetlands: their definition, delineation, and classification for research, sustainable management, and protection. Aquatic Conservation 24(1): 5-22. DOI: http://dx.doi. org/10.1002/aqc.2386

Landgrave, R. y P. Moreno-Casasola. 2012. Evaluación cuantitativa de la pérdida de humedales en México. Investigación Ambiental 4(1): 19-35.

Liang, J., J. Buongiorno, R. A. Monserud, E. L. Kruger y M. Zhou. 2007. Effects of diversity of tree species and size on forest basal area growth, recruitment, and mortality. Forest Ecology and Management 243(1): 116-127. DOI: http://dx.doi.org/10.1016/j.foreco.2007.02.028

López Rosas, H., F. López-Barrera, P. Moreno-Casasola, G. Aguirre-León, E. Cázares-Hernández y L. SánchezHigueredo. 2010. Indicators of recovery in a tropical 
freshwater marsh invaded by an African grass. Ecological Restoration 28(3): 324-332. DOI: http://dx.doi. org/10.3368/er.28.3.324

López Rosas, H. y P. Moreno-Casasola. 2012. Invader versus natives: Effects of hydroperiod on competition between hydrophytes in a tropical freshwater marsh. Basic and Applied Ecology 13(1): 40-49. DOI: http://dx.doi. org/10.1016/j.baae.2011.10.004

Manugistics. 1998. Statgraphics Plus. Standard edition. Rockville, Estados Unidos de América.

Marty, J. T. 2005. Effects of cattle grazing on diversity in ephemeral wetlands. Conservation Biology 19(5): 1626-1632. DOI: http://dx.doi.org/10.1111/j.15231739.2005.00198.x

Mejía-Saulés, M. T. y P. D. D. Aranda. 1992. Gramíneas útiles de México. Cuadernos del Instituto de Biología 16: 30-36.

Menghi, M., N. Montani, N. Monaco, M. Herrera y M. Rosa. 2011. Diversidad y producción primaria de un pastizal inundable no pastoreado en la estepa pampeana (Argentina central). Pastos 28(2): 183-200.

M. E. A. 2005. Ecosystems and human well-being: Wetland and water. Synthesis. Millennium Ecosystem Assessment. Disponible en http://www.millenniumassessment.org/documents/document.358.aspx.pdf (consultado julio de 2015).

Miranda, F. y X. Hernández. 1963. Fisiografía y vegetación. Las zonas áridas del centro y noreste de México. In: Xolocotzia Tomo I. Universidad Autónoma Chapingo. Chapingo, México. Pp. 255-272.

Mitsch, W. J., G. Gosselink, C. Anderson y L. Zhang. 2009. Wetland ecosystems. John Wiley and Sons Inc. Nueva York, USA. 285 pp.

Moreno-Casasola, P. y H. López Rosas. 2009. Muestro y análisis de la vegetación de humedales. In: Moreno-Casasola, P. y B. Warner (eds.). Breviario para describir, observar y manejar humedales. Serie Costa Sustentable no1. RAMSAR, Instituto de Ecología, A.C., Comisión Nacional de Áreas Naturales Protegidas (CONANP), US Fish and Wildlife Service, US State Department. Xalapa, México. Pp. 145-167.

Moreno-Casasola, P., E. Cejudo-Espinosa, A. Capistrán-Barra- das, D. Infante-Mata, H. López-Rosas, G. Castillo-Campos y A. Campos-Cascaredo. 2010. Composición florística, diversidad y ecología de humedales herbáceos emergentes en la planicie costera central de Veracruz, México. Boletín de la Sociedad Botánica de México (87): 29-50.

Moreno-Casasola, P., H. López Rosas y K. Rodríguez-Medina. 2014. From tropical wetlands to pastures on the coast of the Gulf of Mexico. Pastos 42(2): 185-217.

Olff, H. y M. E. Ritchie. 1998. Effects of herbivores on grassland plant diversity. Trends in Ecology and Evolution 13: 261-265. DOI: http://dx.doi.org/10.1016/S0169-5347(98)01364-0

Pollock, M. M., R. J. Naiman y T. A. Hanley.1998. Plant species richness in riparian wetlands-a test of biodiversity theory. Ecology 79(1): 94-105. DOI: http://dx.doi.org/10.1890/0 012-9658(1998)079[0094:PSRIRW]2.0.CO;2

Ponnamperuma, F. N. 1972. The chemistry of submerged soils, Advances in Agronomy 24: 29-88. DOI: http://dx.doi. org/10.1016/S0065-2113(08)60633-1

Portilla Ochoa, E. 2005. Transitando hacia el desarrollo sustentable. El caso de Alvarado, Veracruz Mexico. In: MorenoCasasola, P., E. Peresbarbosa y A. Travieso-Bello (eds.). Manejo integral de la zona costera. Estrategias para el manejo integral de la zona costera: un enfoque municipal. Instituto de Ecología, A.C. y Comisión Nacional de Áreas Naturales Protegidas (CONANP)-Gobierno del Estado de Veracruz. Xalapa, México. Pp. 21-54.

Pykälä, J. 2005. Cattle grazing increases plant species richness of most species trait groups in mesic semi-natural grasslands. Plant Ecology 175(2): 217-226. DOI: http://dx.doi. org/10.1007/s11258-005-0015-y

Richardson, J. L. y M. J. Vepraskas. 2001. Wetland soils. Genesis, Hydrology, Landscapes and Classification. Lewis Publisher. Boca Ratón, USA. 321 pp.

Rodríguez-Medina, K. y P. Moreno-Casasola. 2013. Effect of livestock on soil structure and chemistry in the coastal marshes of the central Gulf Coast of Mexico. Australian Journal of Soil Research 51(4): 341-349. DOI: http://dx. doi.org/10.1071/SR13037

Rosen, D. J., S. L. Hatch y R. Carter. 2008. Taxonomy and nomenclature of three closely related species of Eleocharis subgenus Limnochloa (Cyperaceae). Blumea-Biodiversi- 
ty, Evolution and Biogeography of Plants 53(2): 235-246.

DOI: http://dx.doi.org/10.3767/000651908X607936

Rzedowski, J. 2006. Vegetación de México. Primera edición digital. Comisión Nacional para el Conocimiento y Uso de la Biodiversidad. México, D.F., México. 504 pp.

Travieso-Bello, A., P. Moreno-Casasola y A. Campos. 2005. Efecto de diferentes manejos pecuarios sobre el suelo y la vegetación en humedales transformados a pastizales. Interciencia 30(1): 12-18. http://www.interciencia.org/ v30_01/travieso.pdf (consultado julio de 2015).

Trought, M. C. T. y M. C. Drew. 1980. The development of waterlogging damage in wheat seedlings (Triticum aestivum L.). Plant Soil 54(1): 77-94. DOI: http://dx.doi. org/10.1007/BF02182001

Unger, P. W. y T. C. Kaspar. 1994. Soil compaction and root growth: a review. Agronomy Journal 86(5): 759-766.

DOI: http://dx.doi.org/10.2134/agronj1994.0002196200 $8600050004 \mathrm{x}$

Verdú, J. R., M. B. Crespo y E. Galante. 2000. Conservation strategy of a nature reserve in Mediterranean ecosystems: the effects of protection from grazing on biodiversity.
Biodiversity Conservation 9(12): 1707-1721. DOI: http:// dx.doi.org/10.1023/A:1026506725251

Watkinson, A. y S. Ormerod. 2001. Grasslands, grazing and biodiversity: editors' introduction. Journal of Applied Ecology 38(2): 233-237. DOI: http://dx.doi.org/10.1046/ j.1365-2664.2001.00621.x

Weiher, E. 2003. Species richness along multiple gradients: testing a general multivariate model in oak savannas. Oikos 101(2): 311-316. DOI: http://dx.doi.org/10.1034/ j.1600-0706.2003.12216.x

Westhoff, V. y E. Van der Maarel. 1978. The Braun-Blanquet approach. In: Whittaker, R. H. (ed.). Classification of Plant Communities. 2a ed. Junk. El Haya. Países Bajos. pp. 287-399. DOI: http://dx.doi.org/10.1007/978-94-0099183-5_9

Whittaker, R. H. 1972. Evolution and measurement of species diversity. Taxon 21(2): 213-251. DOI: http://dx.doi. org/10.2307/1218190

Zar, J. H. 1999. Biostatistical Analysis. Prentice Hall. New Jersey, USA. 663 pp. 
Acta Botanica Mexicana 119: 79-99 Abril 2017

Anexo: Listado de las especies encontradas en las localidades de Río Blanco y Río Limón en el municipio de Alvarado, Veracruz, México.

\begin{tabular}{|c|c|c|c|c|c|c|}
\hline Familia & Especie & RB1 & RB2 & RL1 & RL2 & Estatus \\
\hline Alismataceae & Sagittaria lancifolia $\mathrm{L}$. & $\bar{X}$ & $\mathrm{X}$ & $\mathrm{X}$ & $\mathrm{X}$ & Nativa \\
\hline Amaranthaceae & Gomphrena hispida L. & & & & $\mathrm{X}$ & Exótica \\
\hline Araliaceae & Hydrocotyle verticillata Thunb. & $\mathrm{X}$ & & & & Nativa \\
\hline Asteraceae & Ambrosia psilostachya DC. & & & & $\mathrm{X}$ & Nativa \\
\hline \multirow[t]{2}{*}{ Cucurbitaceae } & Cucumis anguria $\mathrm{L}$. & & & & $\mathrm{X}$ & Exótica naturalizada \\
\hline & Momordica charantia $\mathrm{L}$. & & & & $\mathrm{X}$ & Exótica invasiva \\
\hline \multirow[t]{2}{*}{ Cyperaceae } & Cyperus articulatus $\mathrm{L}$. & $\mathrm{X}$ & & $\mathrm{X}$ & & Nativa \\
\hline & Eleocharis cellulosa Torr. & $\mathrm{X}$ & $\mathrm{X}$ & $\mathrm{X}$ & $\mathrm{X}$ & Nativa \\
\hline Euphorbiaceae & Caperonia palustris (L.) A. St.-Hil. & & & & $\mathrm{X}$ & Nativa \\
\hline Fabaceae & Acacia cornigera (L.) Willd & $\mathrm{X}$ & $\mathrm{X}$ & & $\mathrm{X}$ & Nativa \\
\hline Lamiaceae & Leonotis nepetifolia (L.) R. Br. & & & & $\mathrm{X}$ & Exótica \\
\hline Lemnaceae & Lemna minor L. & & $\mathrm{X}$ & & & Nativa \\
\hline Marantaceae & Thalia geniculata $\mathrm{L}$. & & $\mathrm{X}$ & & $\mathrm{X}$ & Nativa \\
\hline Marsileaceae & Marsilea polycarpa Hook. \& Grev & & & & $\mathrm{X}$ & Nativa \\
\hline Nymphaeaceae & Nymphaea ampla (Salisb.) DC. & & $\mathrm{X}$ & $\mathrm{X}$ & & Nativa \\
\hline Onagraceae & Ludwigia octovalvis (Jacq.) Raven & & & & $\mathrm{X}$ & Nativa \\
\hline Plantaginaceae & Bacopa monnieri (L.)Wettst. & $\mathrm{X}$ & $\mathrm{X}$ & $\mathrm{X}$ & & Nativa \\
\hline \multirow[t]{7}{*}{ Poaceae } & Echinochloa colona (L.) Link & $\mathrm{X}$ & $\mathrm{X}$ & & & Exótica \\
\hline & Hymenachne amplexicaulis (Rudge) Nees & & & $\mathrm{X}$ & $\mathrm{X}$ & Nativa invasiva \\
\hline & Leersia hexandra G.C. Tucker & & & $\mathrm{X}$ & & Nativa invasiva \\
\hline & Panicum sp. & & & & $\mathrm{X}$ & -------- \\
\hline & Paspalum sp. & & & & $\mathrm{X}$ & --------- \\
\hline & Sporobolus virginicus (L.) Kunth & $\mathrm{X}$ & & & & Nativa \\
\hline & Urochloa fasciculata Kunth & & & & $\mathrm{X}$ & Nativa \\
\hline Polygonaceae & Polygonum sp. & & & & $\mathrm{X}$ & --------- \\
\hline Pontederiaceae & Pontederia sagittata C. Presl & & $\mathrm{X}$ & $\mathrm{X}$ & $\mathrm{X}$ & Nativa \\
\hline Salviniaceae & Salvinia minima Baker & & $\mathrm{X}$ & & & Exótica invasiva \\
\hline Solanaceae & Solanum campechiense L. & & & $\mathrm{X}$ & $\mathrm{X}$ & Nativa \\
\hline Verbenaceae & Lippia nodiflora (L.) Michx & $\mathrm{X}$ & & & & Nativa \\
\hline Total especies & 29 & 9 & 10 & 9 & 19 & \\
\hline
\end{tabular}

